J. Appl. Numer. Optim. 3 (2021), No. 3, pp. 457-478

Available online at http://jano.biemdas.com

https://doi.org/10.23952/jano.3.2021.3.02

\title{
PHASE RETRIEVAL WITH SUB-GAUSSIAN MEASUREMENTS VIA RIEMANNIAN OPTIMIZATION
}

\author{
HUIPING LI, YU XIA* \\ School of Mathematics, Hangzhou Normal University, Hangzhou 311121, China
}

\begin{abstract}
This paper concerns the phase retrieval problem under random sub-Gaussian measurements. We propose one type of gradient descent method based on Riemannian optimization, namely, truncated Riemannian gradient descent algorithm (TRGrad), to deal with the sub-gaussian phase retrieval problem. Compared with traditional methods, the careful selection rule in our work ensures a tighter initial guess. The sequence generated by the TRGrad converges to the true solution $\boldsymbol{x} \in \mathbb{R}^{n}$ at a geometric rate with high probability provided that the number of measurements $m=O(n)$. This implies that the sample complexity is optimal. In addition, several numerical experiments are provided to show the effectiveness and stability of the TRGrad, and demonstrates that the TRGrad performs better than the state-of-the-art methods, such as Wirtinger Flow (WF) algorithm, and Generalized WF algorithm.
\end{abstract}

Keywords. Gradient descent method; Phase retrieval; Riemannian optimization; Sub-Gaussian measurements.

\section{INTRODUCTION}

1.1. Problem setting. The problem of Phase Retrieval (PR) is generally stated as the problem of estimating a vector $\boldsymbol{x} \in \mathbb{R}^{n}$ or $\mathbb{C}^{n}$ from the certain measurements of the form

$$
\boldsymbol{y}=|\boldsymbol{A x}|^{2}
$$

where $\boldsymbol{y} \in \mathbb{R}^{m}$ is the observation, and $\boldsymbol{A} \in \mathbb{R}^{m \times n}$ is the sensing matrix. The notation $|\cdot|^{2}$ is denoted as:

$$
|z|^{2}=\left[\left|z_{1}\right|^{2}, \ldots,\left|z_{m}\right|^{2}\right]^{\top} \text { for } z=\left[z_{1}, \ldots, z_{m}\right]^{\top} \in \mathbb{R}^{n} .
$$

The above phase retrieval problem has important applications in imaging, optics, signal processing, and communication.

Throughout the paper, the measurement matrix $\boldsymbol{A}$ is drawn from the sub-Gaussian distribution, which is widely used in applications $[1,2,3]$. Before going further, we give the definition of sub-Gaussian random variables.

Definition 1.1. (Sub-Gaussian random variables, [4, 5]) A random variable $\phi$ is called a subGaussian random variable if there exists constants $\kappa>0$ such that $\mathbb{P}(|\phi| \geq t) \leq \exp \left(1-\kappa t^{2}\right)$

${ }^{*}$ Corresponding author.

E-mail addresses: huipingli@hznu.edu.cn (H. Li), yxia@hznu.edu.cn (Y. Xia).

Received October 7, 2021; Accepted November 10, 2021.

(C)2021 Journal of Applied and Numerical Optimization 
for all $t>0$. The sub-Gaussian norm of $\phi$ is denoted as

$$
\|\phi\|_{\psi_{2}}=\sup _{p \geq 1} \frac{\left(\mathbb{E}|\phi|^{p}\right)^{1 / p}}{\sqrt{p}} .
$$

Now we make the explicit definition of sub-Gaussian measurements used in our paper.

Definition 1.2. (sub-Gaussian measurements) Let $\boldsymbol{A} \in \mathbb{R}^{m \times n}$ be the measurements matrix. The elements of $\boldsymbol{A}$ are independently copies of some sub-Gaussian random variable $a$, which meets the conditions as below:

(1) $\mathbb{E} a=0, \mathbb{E} a^{2}=1$;

(2) $\mathbb{E} a^{4}=\tau>1$;

(3) $\|a\|_{\psi_{2}} \leq K(K \geq 1)$.

Remark 1.1. For general sub-Gaussian matrix $\boldsymbol{A}$, we can normalize the elements to meet the condition $\mathbb{E} a^{2}=1$. The forth moment condition $\mathbb{E} a^{4}>1$ is necessary, otherwise the expectation of the concentration inequalities may be smaller than 0 . More details can be seen in Remark 5.1. Based on the definition of $\|\cdot\|_{\psi_{2}}$, if $\|a\|_{\psi_{2}} \leq K$, one has $\tau \leq 16 K^{4}$, and

$$
\mathbb{P}(|a|>t) \leq \exp \left(1-c_{0} t^{2} / K^{2}\right),
$$

where $c_{0}>0$ is some absolute constant.

For simplicity, we focus on the reconstruction of real signal $\boldsymbol{x}$ from the PR problem in this paper. Based on a standard lifting of the signal $\boldsymbol{x}$ to $\boldsymbol{X}=\boldsymbol{x} \boldsymbol{x}^{\top}$, the quadratic measurements (1.1) can be expressed as

$$
y=\mathscr{A}(\boldsymbol{X})
$$

where $\mathscr{A}: \mathbb{R}^{n \times n} \rightarrow \mathbb{R}^{m}$ is defined as below

$$
\mathscr{A}(\boldsymbol{W})=\left\{\left\langle\boldsymbol{W}, \boldsymbol{a}_{j} \boldsymbol{a}_{j}^{\top}\right\rangle\right\}_{j=1}^{m},
$$

with $\boldsymbol{a}_{j}^{\top}$ as the $j$-th row of $\boldsymbol{A}$. Thus, our goal is to estimate the rank-one, and positive semidefinite matrix $\boldsymbol{X}$ from the measurements (1.3), which also solves the PR problem and provides an estimate for the real signal $\boldsymbol{x}$ up to the inevitable global phase ambiguity.

1.2. Notations. For $n \in \mathbb{N}_{+}$, denote $[n]=\{1, \ldots, n\}$. Given and index set $T \subset[n], T^{c}$ is the complement of $T$ in $[n]$. Denote $c_{\alpha}$ or $C_{\alpha}$ as constant depending on parameter $\alpha$. For example, $C_{K}$ depends on parameter $K$, and $C_{K, \tau}$ depends on $K$ and $\tau$. To be convenient, $C$ and $c$ are absolute constants, which may not be the same in different theorems. $A \gtrsim B$ is denoted as $A \geq C_{0} B$, where $C_{0}$ is an absolute positive constant. The notation $\lesssim$ can be defined similarly. $\operatorname{sign}(x)$ is denoted as

$$
\operatorname{sign}(x)= \begin{cases}1 & x \geq 0, \\ -1 & x<0 .\end{cases}
$$

For a vector $\boldsymbol{x} \in \mathbb{R}^{n}$, denote $\|\boldsymbol{x}\|_{p}=\left(\sum_{j=1}^{n}\left|x_{j}\right|^{p}\right)^{1 / p}$ as the $p$-th $(1 \leq p<\infty)$ norm of $\boldsymbol{x}$. $\mathbb{S}^{n-1}$ is the unit $\ell_{2}$-norm sphere in $\mathbb{R}^{n}$. For a matrix $\boldsymbol{X} \in \mathbb{R}^{m \times n}$, write $\boldsymbol{X}^{\top}$ as the transpose of $\boldsymbol{X}$. For any $\boldsymbol{X}, \boldsymbol{Y} \in \mathbb{R}^{n}$, set $\langle\boldsymbol{X}, \boldsymbol{Y}\rangle:=\operatorname{Tr}\left(\boldsymbol{X}^{T} \boldsymbol{Y}\right) . \boldsymbol{X}_{i, j}$ is considered as the $i$-th row and $j$-th column element of $\boldsymbol{X}$. If $\boldsymbol{x}=\left[x_{1}, \ldots, x_{n}\right]^{\top} \in \mathbb{R}^{n}$, then $\operatorname{Diag}(\boldsymbol{x}) \in \mathbb{R}^{n \times n}$ is a diagonal matrix with $[\operatorname{Diag}(\boldsymbol{x})]_{i, i}=x_{i}$. If $\boldsymbol{X} \in \mathbb{R}^{n \times n}$ with Diagonal elements $\left(X_{i, i}\right)_{i=1}^{n}$, then $\operatorname{Diag}(\boldsymbol{X})=\operatorname{Diag}\left(\left[X_{1,1}, \ldots, X_{n, n}\right]^{\top}\right)$. 
1.3. Our contributions. The main challenge in the PR problem under random sub-Gaussian measurements in its general formulation is to design an accurate and computationally tractable estimator that has optimal sample complexity. In this paper, we try to address this challenge by Riemannian optimization. Namely, we propose one kind of truncated Riemannian gradient descent algorithm that

(i) generates a sequence of successive iterates which provably converges to the solution at a geometric rate in the noiseless case;

(ii) the sample complexity is optimal, namely, $m=O(n)$.

Specifically, to confirm our results, we are greatly inspired by the works in $[6,7]$ and attempt to establish a property similar to the restricted isometry property (RIP) and the restricted weak correlation property (RWC) about the sensing operator. However, the linear operator considered here is composed of sub-Gaussian measurements which is more complicated that random Gaussian measurements. For example, the expectation of some complicated functions based on sub-Gaussian vector can not be calculated directly like Gaussian vectors. Therefore, to overcome such difficulties, we need delicate truncation rules and novel update rules to produce a tighter initial guess, better descent directions, and thus enhanced practical performance. This implies that our work is substantially different from that in [6]. Thus, it is by no means trivial to establish the convergence for sub-Gaussian measurements. Our analyses rely on several key technical lemmas, namely, Lemma 3.1 and Lemma 3.2.

1.4. Related works. As is well known, the PR problem arises in many areas of science and engineering such as in X-ray crystallography, astronomy, quantum mechanics, and diffraction imaging; see, e.g., $[8,9,10,11,12,13]$. Because of the practical significance of the phase retrieval problem in different areas, the community has developed a large body of methods for recovering a signal from the PR problem. For the classical PR problem, which focuses on the Fourier transform, the most widely used method are perhaps Gerchberg-Saxton algorithm [14] and Fienup algorithm [15]. While such methods perform well in practice, the fundamental mathematical questions concerning their convergence remain unresolved. Recently, a family of algorithms based on convex or non-convex optimizations have been developed to solve the general PR problem under different measurements. We refer the reader to [16] for a recent review. The most popular convex method is the perhaps PhaseLift algorithm proposed in [17, 18, 19] and for Gaussian or sub-Gaussian measurements, the PhaseLift method is proved to provide exact solutions for the general PR problem using an optimal number of sampling vectors $[2,20]$. While such method based on SDP relaxations offer tractable solutions, it becomes computationally prohibitive as the dimension of the signal increases. As a result, many nonconvex algorithms have also been provided to directly solve the phase retrieval problem in the original signal space; see, e.g., [1, 21, 22, 23, 24, 25]. For the theoretical side, the generalized WF algorithm is demonstrated to produce a good estimator for the sub-Gaussian PR problem, provided that the number of measurements $m=O\left(n \log ^{2} n\right)$ in [1]. Besides, to the best of our knowledge, there are few algorithms with theoretical guarantees established for PR problem in the case of random sub-Gaussian measurements. 


\section{TRunCATEd Riemannian Gradient Descent Algorithm}

In this section, we establish the truncated Riemannian gradient descent algorithm for subGaussian phase retrieval problem. The truncated Riemannian gradient descent algorithm (TRGrad) in Algorithm 1 exploits the embedded manifold of rank-1 and positive semidefinite matrices.

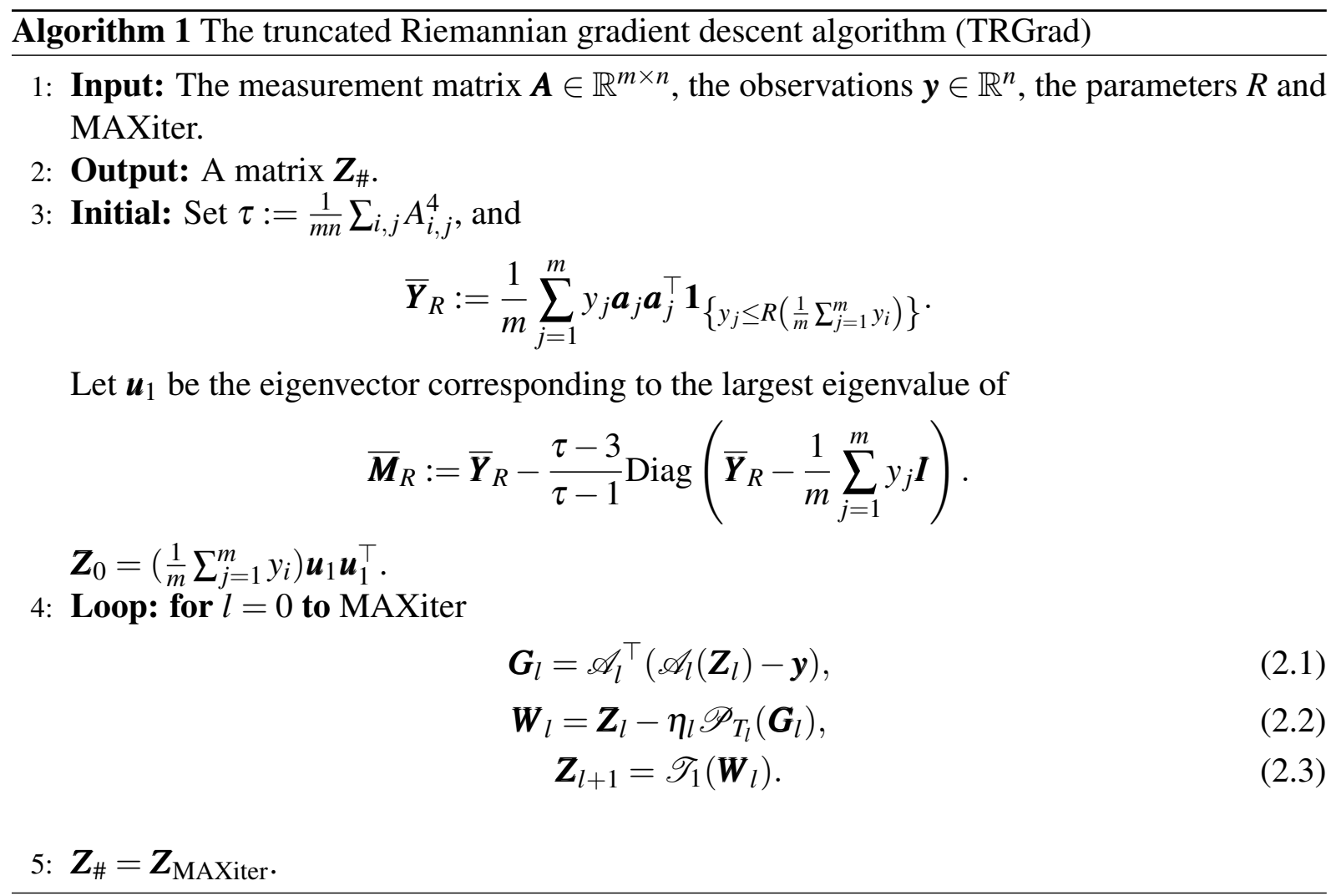

The estimation of forth moment $\tau$ depends on the law of large numbers since $Z_{l}$ is rank-1 and positive semidefinite with

$$
\mathbf{Z}_{l}=\boldsymbol{z}_{l} \boldsymbol{z}_{l}^{\top}
$$

In (2.1), $\boldsymbol{A}_{l}$ is some linear operator defined as

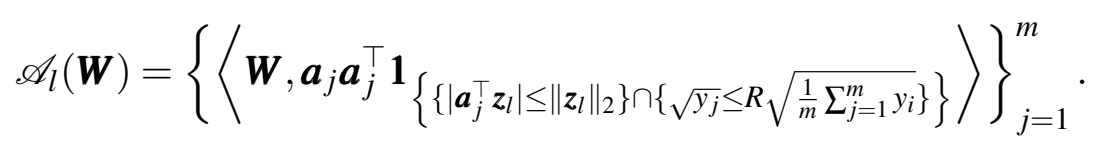

Then the adjoint of $\mathscr{A}_{l}$ is given by

$$
\mathscr{A}_{l}^{\top}(\boldsymbol{b})=\sum_{j=1}^{m} b_{j} \boldsymbol{a}_{j} \boldsymbol{a}_{j}^{\top} \mathbf{1}_{\left\{\left\{\left|\boldsymbol{a}_{j}^{\top} \boldsymbol{z}_{l}\right| \leq\left\|\boldsymbol{z}_{l}\right\|_{2}\right\} \cap\left\{\sqrt{y_{j}} \leq R \sqrt{\frac{1}{m} \sum_{j=1}^{m} y_{i}}\right\}\right.},
$$

for all $\boldsymbol{b} \in \mathbb{R}^{m}$. The projection $\mathscr{P}_{T_{l}}$ in (2.2) can be computed as follows:

$$
\mathscr{P}_{T_{l}}(\boldsymbol{W})=\boldsymbol{u}_{l} \boldsymbol{u}_{l}^{\top} \boldsymbol{W}+\boldsymbol{W} \boldsymbol{u}_{l} \boldsymbol{u}_{l}^{\top}-\boldsymbol{u}_{l} \boldsymbol{u}_{l}^{\top} \boldsymbol{W} \boldsymbol{u}_{l} \boldsymbol{u}_{l}^{\top},
$$

where $\boldsymbol{u}_{l}=z_{l} /\left\|z_{l}\right\|_{2}$. 
In (2.3), $\mathscr{P}_{T_{1}}$ returns the best rank-1 and positive semidefinite approximation of $W_{l}$. Since $\boldsymbol{W}_{l}=\boldsymbol{W}_{l}^{\top}$ and the rank of $\boldsymbol{W}_{l}$ is no larger than 2 , the eigenvalue decomposition of $\boldsymbol{W}_{l}$ can be constructed easily.

\section{Main Results}

In this section, we first show the convergence result on the TRGrad by considering general measurement matrix $\boldsymbol{A}$. For any $\boldsymbol{z} \in \mathbb{R}^{n}$, denote

$$
\begin{aligned}
& T_{z}=\left\{\boldsymbol{z} \boldsymbol{w}^{\top}+\boldsymbol{w} \boldsymbol{z}^{\top} \mid \boldsymbol{w} \in \mathbb{R}^{n}\right\},
\end{aligned}
$$

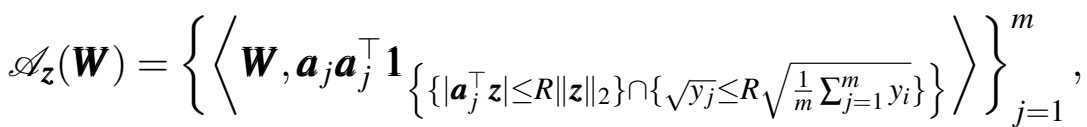

and

$$
\mathscr{P}_{z}(\boldsymbol{W})=\boldsymbol{u} \boldsymbol{u}^{\top} \boldsymbol{W}+\boldsymbol{W} \boldsymbol{u} \boldsymbol{u}^{\top}-\boldsymbol{u} \boldsymbol{u}^{\top} \boldsymbol{W} \boldsymbol{u} \boldsymbol{u}^{\top}
$$

with $\boldsymbol{u}=\boldsymbol{z} /\|\boldsymbol{z}\|_{2}$.

We propose two important local conditions as below.

Condition 1: Truncated Local Restricted Isometry Property(TL-RIP $(\alpha, \beta))$.

The measurement matrix $\boldsymbol{A}$ satisfies the Truncated Local Restricted Isometry Property with parameters $\alpha, \beta>0$ if, for all $z z^{\top}$ obeying $\left\|z z^{\top}-\boldsymbol{x} \boldsymbol{x}^{\top}\right\|_{F} \leq \varepsilon_{0}\left\|\boldsymbol{x} \boldsymbol{x}^{\top}\right\|_{F}$ and $\boldsymbol{W} \in T_{z}$,

$$
\alpha\|\boldsymbol{W}\|_{F}^{2} \leq \frac{1}{m}\left\|\mathscr{A}_{z}(\boldsymbol{W})\right\|_{2}^{2} \leq \beta\|\boldsymbol{W}\|_{F}^{2} .
$$

Condition 2: Truncated Local Weak Correlation Property(TL-WCP( $\theta)$ ).

The measurement matrix $\boldsymbol{A}$ satisfies the Truncated Local Weak Correlation Property with parameter $\theta>0$ if, for all $\boldsymbol{z} \boldsymbol{z}^{\top}$ obeying $\left\|\boldsymbol{z} \boldsymbol{z}^{\top}-\boldsymbol{x} \boldsymbol{x}^{\top}\right\|_{F} \leq \boldsymbol{\varepsilon}_{0}\left\|\boldsymbol{x} \boldsymbol{x}^{\top}\right\|_{F}$ and $\boldsymbol{W} \in T_{\boldsymbol{z}}$,

$$
\frac{1}{m}\left\|\mathscr{P}_{T_{z}} \mathscr{A}_{z}^{\top} \mathscr{A}_{z}\left(\boldsymbol{I}-\mathscr{P}_{T_{z}}\right)\left(\boldsymbol{z} \boldsymbol{z}^{\top}-\boldsymbol{x} \boldsymbol{x}^{\top}\right)\right\|_{F} \leq \theta\left\|\boldsymbol{z} \boldsymbol{z}^{\top}-\boldsymbol{x} \boldsymbol{x}^{\top}\right\|_{F} .
$$

Theorem 3.1 relies on $\operatorname{TL}-\operatorname{RIP}(\alpha, \beta)$ and $\operatorname{TL}-\mathrm{WCP}(\theta)$.

Theorem 3.1. (General convergence result) Denote $X=\boldsymbol{x} \boldsymbol{x}^{\top}$. Assume that the initial guess $\boldsymbol{Z}_{0}=z_{0} z_{0}^{\top}$ satisfies $\left\|z_{0} z_{0}^{\top}-\boldsymbol{x} \boldsymbol{x}^{\top}\right\|_{F} \leq \varepsilon_{0}\|\boldsymbol{X}\|_{F}$. If the measurement matrix $\boldsymbol{A}$ satisfies $T L$ $\operatorname{RIP}(\alpha, \beta)$ and $T L-W C P(\theta)$, then

$$
\left\|Z_{l+1}-\boldsymbol{X}\right\|_{F} \leq v\left\|Z_{l}-\boldsymbol{X}\right\|_{F},
$$

for some $0<v<1$. The step-size $\eta_{l}$ is taken as

$$
\eta_{l} \in\left[\frac{1-1 / \sqrt{1+16 \varepsilon_{0}^{2}}+\varepsilon_{0}}{\alpha-\theta}, \frac{1+1 / \sqrt{1+16 \varepsilon_{0}^{2}}-\varepsilon_{0}}{\beta+\theta}\right]
$$

provided that

$$
\theta<\alpha \text { and }\left(1-1 / \sqrt{1+16 \varepsilon_{0}^{2}}+\varepsilon_{0}\right) \beta+2 \theta \leq\left(1+1 / \sqrt{1+16 \varepsilon_{0}^{2}}-\varepsilon_{0}\right) \alpha
$$

When $\varepsilon_{0}$ and $\theta$ is small enough, the condition in (3.1) can be satisfied easily.

Now we focus on sub-Gaussian measurements drawn from distribution in Definition 1.2 to show that the conditions in Theorem 3.1 can be achieved with optimal sampling complexity. 
Theorem 3.2. (Initialization result on sub-Gaussian measurements) Assume that the elements of $\boldsymbol{A} \in \mathbb{R}^{m \times n}$ are independently copies of sub-Gaussian random variable a in Definition 1.2 with parameters $K$ and $\tau . Z_{0}$ is the initialization generated as in Algorithm 1, if $m \geq C_{\delta, K, \tau} n$, then

$$
\left\|\boldsymbol{Z}_{0}-\boldsymbol{X}\right\|_{F} \leq \sqrt{5 \delta}(\sqrt{5 \delta}+2)\|\boldsymbol{X}\|_{F}
$$

with probability at least $1-\exp (-\underline{c} n)$.

The following two lemmas demonstrate the TL-RIP and TL-WCP on sub-Gaussian measurements.

Lemma 3.1. The elements of $\boldsymbol{A} \in \mathbb{R}^{m \times n}$ are independently copies of sub-Gaussian random variable $a$ in Definition 1.2 with parameters $K$ and $\tau>1$. $\boldsymbol{a}_{j}^{\top}$ is the $j$-th row of $\boldsymbol{A}$. Then with probability at least $1-\exp \left(-c_{K} m\right)$, we have

$$
\min \left\{\frac{1}{8}, \frac{\tau-1}{16}\right\}\|\boldsymbol{W}\|_{F}^{2} \leq \frac{1}{m}\left\|\mathscr{A}_{z}(\boldsymbol{W})\right\|_{2}^{2} \leq \frac{3+2|\tau-3|}{2}\|\boldsymbol{W}\|_{F}^{2}
$$

holds uniformly for all $\boldsymbol{z} \in \mathbb{R}^{n}$ and all $\boldsymbol{W}=\boldsymbol{z} \boldsymbol{w}^{\top}+\boldsymbol{w} \boldsymbol{z}^{\top}$ provided that $m \geq C_{K} n$.

Lemma 3.2. The elements of $\boldsymbol{A} \in \mathbb{R}^{m \times n}$ are independently copies of sub-Gaussian random variable $a$ in Definition 1.2 with parameters $K$ and $\tau>1$. $\boldsymbol{a}_{j}^{\top}$ is the $j$-th row of $A$. Denote $\boldsymbol{a}_{j} \in \mathbb{R}^{n}(j=1, \ldots, m)$ as the row elements of $\boldsymbol{A}$. Then with probability at least $1-\exp \left(-c_{K} m\right)$, we have

$$
\begin{aligned}
& \frac{1}{m}\left\|\mathscr{P}_{T_{z}} \mathscr{A}_{z}^{\top} \mathscr{A}_{\boldsymbol{z}}\left(\boldsymbol{I}-\mathscr{P}_{T_{z}}\right)\left(\boldsymbol{z} \boldsymbol{z}^{\top}-\boldsymbol{x} \boldsymbol{x}^{\top}\right)\right\|_{F} \\
& \leq\left(\sqrt{\frac{3+2|\tau-3|}{2}}\right)\left(\delta+5 R^{4} K^{2} \exp \left(1-\frac{c_{1} R^{2}}{K^{2}}\right)\right)\left\|z z^{\top}-\boldsymbol{x x} \boldsymbol{x}^{\top}\right\|_{F}
\end{aligned}
$$

holds uniformly for all $\boldsymbol{z z ^ { \top }}$ obeying $\left\|\boldsymbol{z} \boldsymbol{z}^{\top}-\boldsymbol{x} \boldsymbol{x}^{\top}\right\| \leq \varepsilon_{0}\|\boldsymbol{X}\|_{F}$ provided that $m \geq C_{K} m$. Here

$$
\varepsilon_{0} \leq \frac{1}{2} \min \left\{\sqrt{\frac{\delta}{2\left(2 R^{4}+5 R^{3}+8 R^{2}\right)}}, \frac{\delta}{32 R}, \frac{1}{2}\right\} .
$$

Combining Lemma 3.1, Lemma 3.2, Theorem 3.2, and Theorem 3.1, and taking constants $\delta$ and $R$ sufficiently small and large, respectively, the following result can be obtained immediately.

Theorem 3.3. (Convergence result on sub-Gaussian measurements) The elements of $\boldsymbol{A} \in \mathbb{R}^{m \times n}$ are independently copies of sub-Gaussian random variable a in Definition 1.2 with parameters $K$ and $\tau>1$. Algorithm 1 generates $\mathbf{Z}_{l}$ such that

$$
\left\|\mathbf{Z}_{l+1}-\boldsymbol{X}\right\|_{F} \leq v\left\|\mathbf{Z}_{l}-\boldsymbol{X}\right\|_{F},
$$

for some $0<v<1$ with probability $1-\exp \left(-c_{K} m\right)$ provided that $m \geq C_{K} n$.

\section{NUMERICAL EXPERIMENTS}

4.1. Experiment settings. In this following numerical experiments, we will investigate the performance of the TRGrad in Algorithm 1 compared with Generalized WF [1] and WF [21]. Here we choose $R=3 \sqrt{\tau}$ in TRGrad. To make the comparison fair, the maximum iterations are 
taken to be the same in different algorithms. All experiments are carried out on Matlab 2018 with a $2.9 \mathrm{GHz}$ Intel Core $\mathrm{i} 7-10700 \mathrm{~F}$ and $16 \mathrm{~GB}$ memory.

The signal dimension $n=128$. The target signal $\boldsymbol{x}=\left[x_{1}, \ldots, x_{n}\right]^{\top}$ is drawn from Gaussian distribution, i.e.,

$$
x_{j}= \begin{cases}\widetilde{x}_{j} & j \leq n-1, \\ 100 \widetilde{x}_{j} & j=n,\end{cases}
$$

where $\widetilde{\boldsymbol{x}}=\left[\widetilde{x}_{1}, \ldots, \widetilde{x}_{n}\right]$ is standard random Gaussian vector with $\widetilde{x}_{j} \sim \mathscr{N}(0,1)$. Besides, two measurement models are considered:

(1) Uniform distribution model: the elements of $A$ are drawn from $\sqrt{3} \mathscr{U}[-1,1]$.

(2) Ternary distribution model: the elements of $\boldsymbol{A}$ are drawn from the following distribution

$$
t= \begin{cases}\sqrt{3 / 2}, & \text { with Prob. } 1 / 3 \\ 0, & \text { with Prob. 1/3 } \\ -\sqrt{3 / 2}, & \text { with Prob. 1/3. }\end{cases}
$$

If the algorithm output is $\boldsymbol{Z}_{\#}=\boldsymbol{z}_{\#} \boldsymbol{Z}_{\#}^{\top}$, we obtain the performance in terms of relative mean square error (MSE), that is,

where

$$
\text { Relative MSE }:=\frac{\operatorname{dist}\left(\boldsymbol{z}_{\#}, \boldsymbol{x}\right)}{\|\boldsymbol{x}\|_{2}}
$$

$$
\operatorname{dist}\left(z_{\#}, \boldsymbol{x}\right)=\min \left\{\left\|z_{\#}-\boldsymbol{x}\right\|_{2},\left\|z_{\#}+\boldsymbol{x}\right\|_{2}\right\} .
$$

We consider an algorithm to have successfully reconstructed a target signal $x$ if the relative MSE is smaller than $10^{-5}$.

4.2. Numerical performance. First of all, we investigate the initialization step of our algorithm TRGrad compared with WF and generalized WF. The sampling ratio $m / n$ ranges from 2 to 10 . For each $m / n$, we repeat the experiments for 50 times and show the average value of the relative error. Figure 4.1 depicts the relative error versus $m / n$ for uniform distribution model and ternary distribution model. We find that the relative error by Generalized WF and TRGrad is smaller compared with that by WF. However, the performance on initialization is comparable between Generalized WF and TRGrad. Since the sampling complexity is optimal in TRGrad, we believe that with more delicate proofs, the sampling complexity can be reduce in generalized WF [1].

Then we test the success rate under different algorithms. We set the maximal iteration number as 1000 and evaluate the algorithms by 50 trials. The plots of successful recovery probability against the sampling ratio $m / n$ are shown in Figure 4.2. We find that TRGrad has improved performance over Generalized WF and WF, and 3.5n measurements are enough for exact recovery in both uniform distribution model and ternary distribution model. To discuss the computational efficiency, we consider the convergence time on successful recovery. Table 1 shows the average running time in 50 tests when $m / n$ ranges from 5 to 8 . Since the maximal iteration number is fixed, it also includes the unsuccessful recovery examples in WF (the relative MSE may still be larger than $10^{-5}$ when iteration ends). On one hand, TRGrad exhibits a superior performance under two measurement models. On the other hand, the computational time on successfully recover signal may decrease even when the sampling ratio $m / n$ increases. Besides, we also 


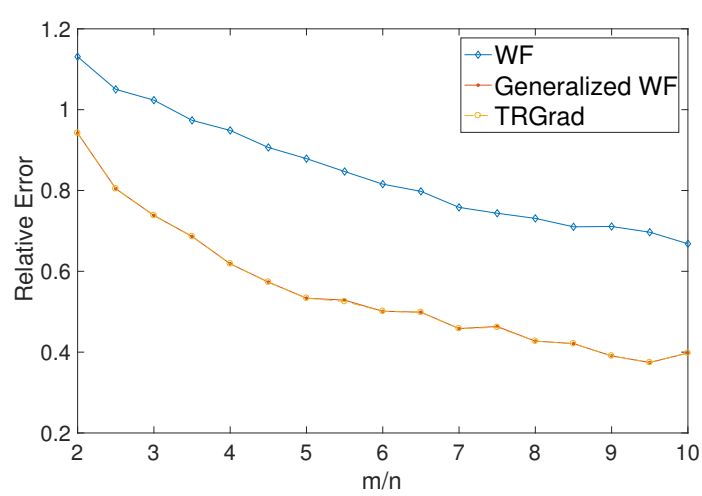

(A)

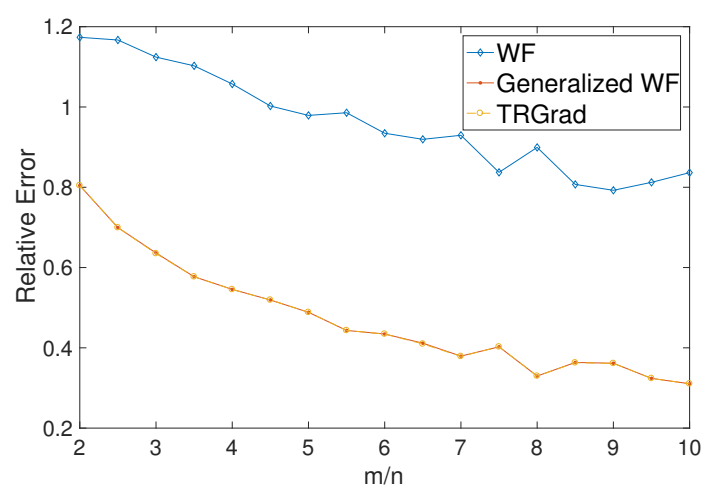

(B)

FIGURE 4.1. Initialization comparison under different measurements: (a) uniform distribution model; (b) ternary distribution model.

compare the convergence rates of different algorithms when $m / n=6$. In Figure 4.3, TRGrad converges much faster than the other two algorithms.

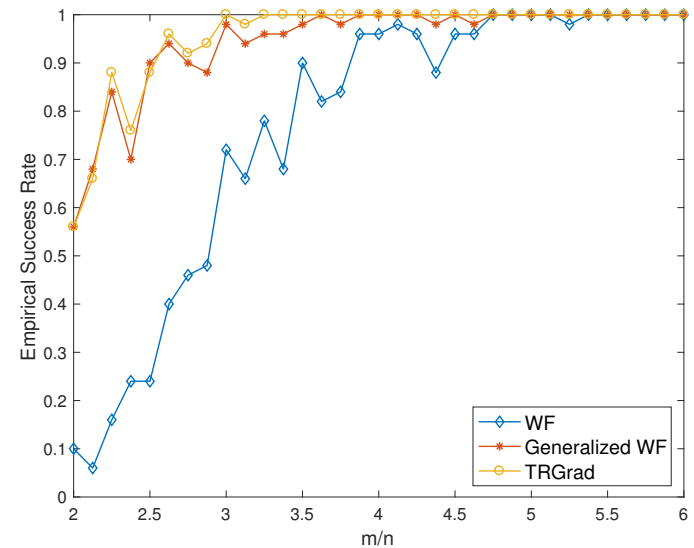

(A)

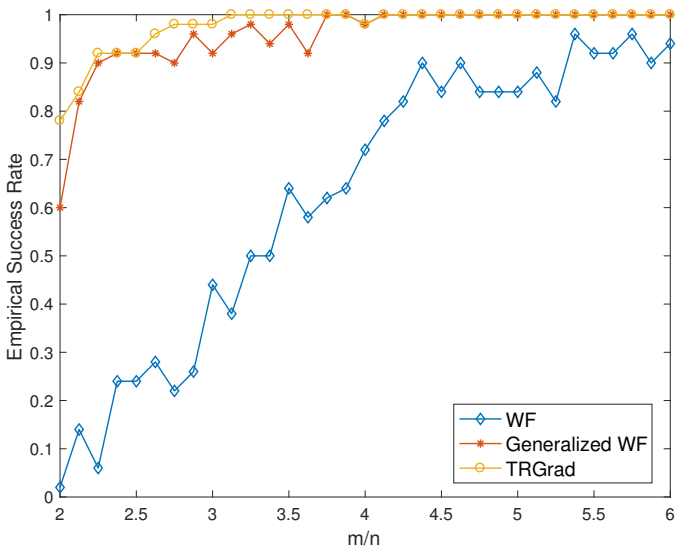

(B)

FIGURE 4.2. Success rate comparison under different measurements: (a) uniform distribution model; (b) ternary distribution model.

Furthermore, we demonstrate the performance on different algorithms under additive noise. The white Gaussian noise is followed by MATLAB function $\operatorname{awgn}\left(\mathscr{A}\left(\boldsymbol{x} \boldsymbol{x}^{\top}\right)\right.$,snr $)$, i.e., $\boldsymbol{y}=$ $|\boldsymbol{A x}|^{2}+\boldsymbol{w}$ with $\boldsymbol{w} \sim \sqrt{\frac{\|\mathscr{A}(\boldsymbol{x})\|_{2}^{2} / m}{10^{\mathrm{snr} / 10}}} \mathscr{N}\left(0, \mathbf{I}_{m}\right)$. Here $m / n=6$ and 50 trials are conducted. The SNR varies from $10 \mathrm{~dB}$ to $50 \mathrm{~dB}$. We compute the signal-to noise ratio of reconstruction in $\mathrm{dB}$ as $-20 \log _{10}$ (relative MSE). The average relative reconstruction error is dB plotted against SNR is shown in Figure 4.4. For TRGrad and Generalized WF, the desirable linear scaling between the noise levels and the relative reconstruction errors can be observed. The WF fails to meet the property since the signal may not be recovered even in noiseless case. Although the relative error is comparable between Generalized WF and TRGrad in noisy case, the convergence rates 
TABLE 1. Average running time (seconds) under different measurements

\begin{tabular}{|c|c||c|c|c|c|}
\hline & sampling ratio $(\mathrm{m} / \mathrm{n})$ & 5 & 6 & 7 & 8 \\
\hline \multirow{3}{*}{ Uniform distribution model } & WF & 0.0577 & 0.0434 & 0.0444 & 0.1325 \\
& Generalized WF & 0.0450 & 0.0347 & 0.0365 & 0.0824 \\
& TRGrad & $\mathbf{0 . 0 2 4 7}$ & $\mathbf{0 . 0 1 8 3}$ & $\mathbf{0 . 0 1 8 5}$ & $\mathbf{0 . 0 3 5 8}$ \\
\hline \multirow{3}{*}{ Ternary distribution model } & WF & 0.0597 & 0.0487 & 0.0524 & 0.1458 \\
& Generalized WF & 0.0349 & 0.0355 & 0.0369 & 0.0829 \\
& TRGrad & $\mathbf{0 . 0 1 9 5}$ & $\mathbf{0 . 0 1 7 6}$ & $\mathbf{0 . 0 1 8 4}$ & $\mathbf{0 . 0 3 5 8}$ \\
\hline
\end{tabular}

are quite different. In Figure 4.5, we find that TRGrad also converges much faster than WF and Generalized WF, when the observations are corrupted by additional noise.

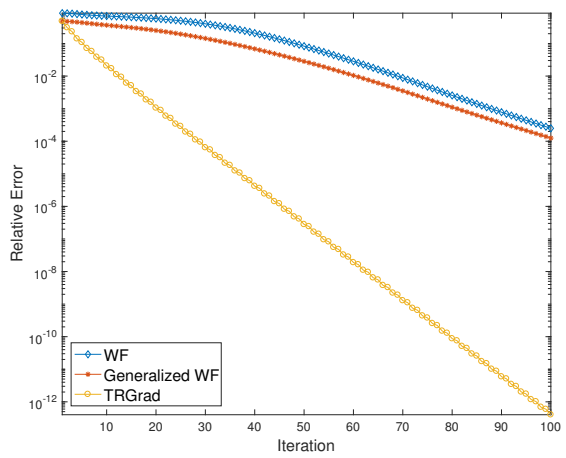

(A)

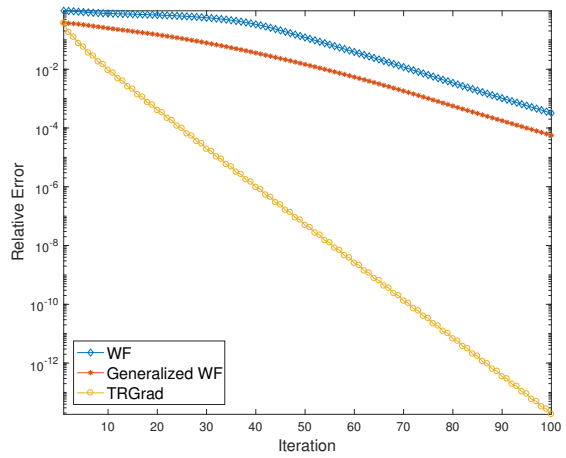

(B)

FIGURE 4.3. Convergence rate comparison under different measurements: (a) uniform distribution model; (b) ternary distribution model.

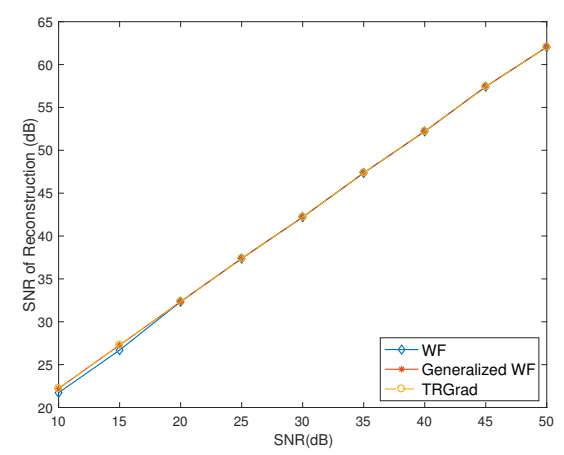

(A)

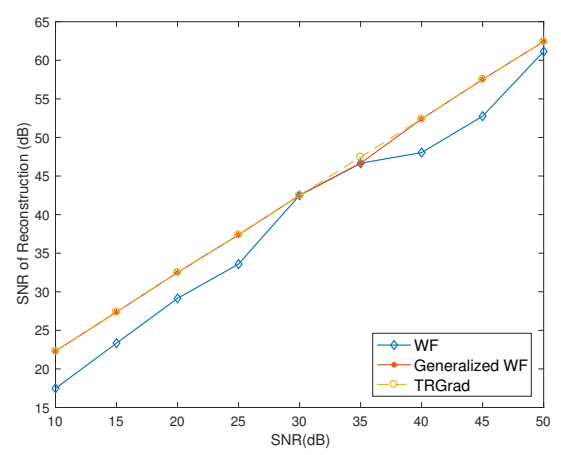

(B)

FIGURE 4.4. Performance under different SNR: (a) uniform distribution model; (b) ternary distribution model. 


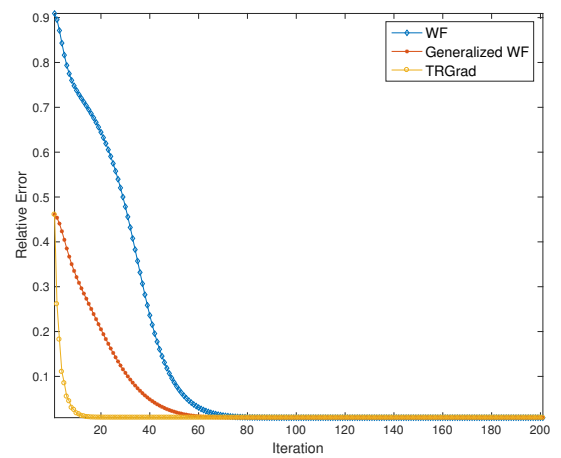

(A)

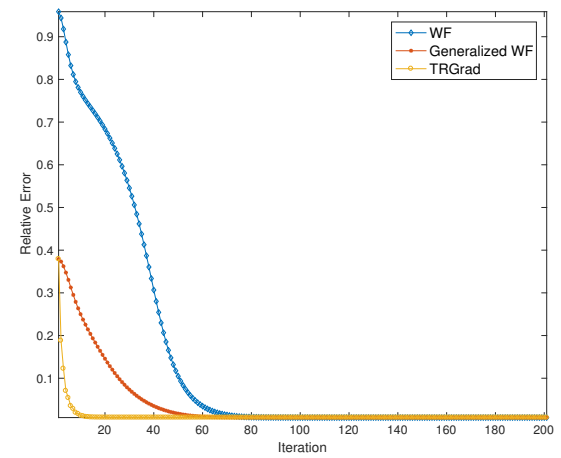

(B)

FIGURE 4.5. Relative MSE against iteration: (a) uniform distribution model; (b) ternary distribution model.

\section{PROOFS}

5.1. The estimation of expectation. We estimate the expectation of $\left(\frac{1}{m} \sum_{i=1}^{m}\left|\left\langle\boldsymbol{x}, \boldsymbol{a}_{j}\right\rangle\right|^{2} \boldsymbol{a}_{j} \boldsymbol{a}_{j}^{\top}\right)$ first and then calculate its upper and lower bounds, which will play important roles in the main results.

Theorem 5.1. Assume that $\boldsymbol{y}=|\boldsymbol{A x}|^{2}$ with $\boldsymbol{A} \in \mathbb{R}^{m \times n}$ and $\boldsymbol{x}=\left[x_{1}, \ldots, x_{n}\right]^{\top}$. The elements of $A \in \mathbb{R}^{m \times n}$ are independently copies of sub-Gaussian random variable a in Definition 1.2 with parameters $K$ and $\tau$. $\boldsymbol{a}_{j}^{\top}$ is the $j$-th row of $\boldsymbol{A}$. Then

$$
\mathbb{E}\left(\frac{1}{m} \sum_{i=1}^{m}\left|\left\langle\boldsymbol{x}, \boldsymbol{a}_{j}\right\rangle\right|^{2} \boldsymbol{a}_{j} \boldsymbol{a}_{j}^{\top}\right)=2 \boldsymbol{x} \boldsymbol{x}^{\top}+\|\boldsymbol{x}\|_{2}^{2} \boldsymbol{I}+(\tau-3) \operatorname{Diag}\left(|\boldsymbol{x}|^{2}\right) .
$$

Proof. Denote $\boldsymbol{a}=\left[a_{1}, \ldots, a_{n}\right]^{\top} \in \mathbb{R}^{n}$, where $a_{i}$ are independently copies of sub-Gaussian random variable $a$ in Definition 1.2. Then

$$
\mathbb{E}\left(\frac{1}{m} \sum_{i=1}^{m}\left|\left\langle\boldsymbol{x}, \boldsymbol{a}_{j}\right\rangle\right|^{2} \boldsymbol{a}_{j} \boldsymbol{a}_{j}^{\top}\right)=\mathbb{E}\left(|\langle\boldsymbol{x}, \boldsymbol{a}\rangle|^{2} \boldsymbol{a} \boldsymbol{a}^{\top}\right) .
$$

By simple calculation, we have

$$
\begin{aligned}
\mathbb{E}\left[\left.\langle\boldsymbol{x}, \boldsymbol{a}\rangle\right|^{2} \boldsymbol{a}^{\top} \boldsymbol{a}\right]_{j, k} & =\mathbb{E}\left(\left(\sum_{i=1}^{n} x_{i} a_{i}\right)^{2} a_{j} a_{k}\right)= \begin{cases}\tau\left|x_{j}\right|^{2}+\sum_{l \neq j}\left|x_{l}\right|^{2}, & j=k, \\
2 x_{j} x_{k}, & j \neq k,\end{cases} \\
& = \begin{cases}2\left|x_{j}\right|^{2}+\|\boldsymbol{x}\|_{2}^{2}+(\tau-3)\left|x_{j}\right|^{2}, & j=k, \\
2 x_{j} x_{k}, & j \neq k,\end{cases}
\end{aligned}
$$

which meets the conclusion.

Lemma 5.1. Let $\tau>1$ be some fixed constant. For any $\boldsymbol{w}, \boldsymbol{x} \in \mathbb{R}^{n}$ such that $\|\boldsymbol{w}\|_{2}=\|\boldsymbol{x}\|_{2}=1$,

$$
0<\min \left\{1, \frac{\tau-1}{2}\right\} \leq \boldsymbol{w}^{\top}\left(2 \boldsymbol{x} \boldsymbol{x}^{\top}+\boldsymbol{I}+(\tau-3) \operatorname{Diag}\left(|\boldsymbol{x}|^{2}\right)\right) \boldsymbol{w} \leq 3+|\tau-3| .
$$


Remark 5.1. The condition that $\tau>1$ can not removed, otherwise, the lower bound may be smaller than 0. For example, take $\boldsymbol{x}=[\sqrt{2} / 2, \sqrt{2} / 2]^{\top}$ and $\boldsymbol{w}=[-\sqrt{2} / 2, \sqrt{2} / 2]^{\top}$. Then

$$
\boldsymbol{w}^{\top}\left(2 \boldsymbol{x} \boldsymbol{x}^{\top}+\boldsymbol{I}+(\tau-3) \operatorname{Diag}\left(|\boldsymbol{x}|^{2}\right)\right) \boldsymbol{w}=1+(\tau-3) / 2<0 .
$$

Proof. Since

$$
\boldsymbol{w}^{\top}\left(2 \boldsymbol{x} \boldsymbol{x}^{\top}+\boldsymbol{I}+(\tau-3) \operatorname{Diag}\left(|\boldsymbol{x}|^{2}\right)\right) \boldsymbol{w}=2|\langle\boldsymbol{x}, \boldsymbol{w}\rangle|^{2}+1+(\tau-3)\left\langle|\boldsymbol{x}|^{2},|\boldsymbol{w}|^{2}\right\rangle,
$$

we need to estimate the upper and lower bound of $2|\langle\boldsymbol{x}, \boldsymbol{w}\rangle|^{2}+1+(\tau-3)\left\langle|\boldsymbol{x}|^{2},|\boldsymbol{w}|^{2}\right\rangle$.

The upper bound can be obtained directly, that is,

$$
2|\langle\boldsymbol{x}, \boldsymbol{w}\rangle|^{2}+1+(\tau-3)\left\langle|\boldsymbol{x}|^{2},|\boldsymbol{w}|^{2}\right\rangle \leq 2+1+|\tau-3|=3+|\tau-3|,
$$

applying Cauchy-Swartcz inequality. Then we should estimate the lower bound of (5.1). If $\tau \geq 3$, then

$$
2|\langle\boldsymbol{x}, \boldsymbol{w}\rangle|^{2}+1+(\tau-3)\left\langle|\boldsymbol{x}|^{2},|\boldsymbol{w}|^{2}\right\rangle \geq 1
$$

Therefore, the only thing left is to calculate the lower bound of (5.1) when $\tau<3$. For any $\boldsymbol{w}=\left[w_{1}, \ldots, w_{n}\right]^{\top}$ and $\boldsymbol{x}=\left[x_{1}, \ldots, x_{n}\right]^{\top}$ with $\|\boldsymbol{w}\|_{2}=\|\boldsymbol{x}\|_{2}=1$, denote

$$
I:=\left\{i \in[n] \mid \operatorname{sign}\left(w_{i}\right)=\operatorname{sign}\left(x_{i}\right)\right\} .
$$

Take $A=\sum_{i \in I} x_{i} w_{i}$ and $B=-\sum_{i \in I^{c}} x_{i} w_{i}$. Since $x_{i} w_{i} \geq 0$ for $i \in I$ and $-x_{i} w_{i} \geq 0$ for $i \in I^{c}$, we have

$$
A \geq 0, B \geq 0, \text { and }|\langle\boldsymbol{x}, \boldsymbol{w}\rangle|^{2}=(A-B)^{2} \text {. }
$$

Then

$$
\begin{aligned}
& 2|\langle\boldsymbol{x}, \boldsymbol{w}\rangle|^{2}+1+(\tau-3)\left\langle|\boldsymbol{x}|^{2},|\boldsymbol{w}|^{2}\right\rangle \\
= & 2(A-B)^{2}+1+(\tau-3)\left(\sum_{i \in I}\left(x_{i} w_{i}\right)^{2}+\sum_{i \in I^{c}}\left(x_{i} w_{i}\right)^{2}\right) \\
\geq & 2(A-B)^{2}+1+(\tau-3)\left(A^{2}+B^{2}\right) \\
\geq & \max \left\{2(A-B)^{2}+(A+B)^{2}+(\tau-3)\left(A^{2}+B^{2}\right), 1+(\tau-3)\left(A^{2}+B^{2}\right)\right\} \\
\geq & \max \left\{(A-B)^{2}+(A+B)^{2}+(\tau-3)\left(A^{2}+B^{2}\right), 1+(\tau-3)\left(A^{2}+B^{2}\right)\right\} \\
= & \max \left\{(\tau-1)\left(A^{2}+B^{2}\right), 1+(\tau-3)\left(A^{2}+B^{2}\right)\right\} .
\end{aligned}
$$

The second line is based on

$$
\sum_{i \in I}\left(x_{i} w_{i}\right)^{2} \leq\left(\sum_{i \in I} x_{i} w_{i}\right)^{2}=A^{2}
$$

and

$$
\sum_{i \in I^{c}}\left(x_{i} w_{i}\right)^{2} \leq\left(\sum_{i \in I^{c}} x_{i} w_{i}\right)^{2}=B^{2}
$$

The third line is based on

$$
A+B \leq \frac{1}{2} \sum_{i \in I}\left(x_{i}^{2}+w_{i}^{2}\right)+\frac{1}{2} \sum_{i \in I^{c}}\left(x_{i}^{2}+w_{i}^{2}\right)=1 .
$$


Therefore, when $\tau<3$, the lower bound can be taken as

$$
\begin{aligned}
& 2|\langle\boldsymbol{x}, \boldsymbol{w}\rangle|^{2}+1+(\tau-3)\left\langle|\boldsymbol{x}|^{2},|\boldsymbol{w}|^{2}\right\rangle \\
& \geq \min _{A^{2}+B^{2} \leq 1}\left(\max \left\{(\tau-1)\left(A^{2}+B^{2}\right), 1+(\tau-3)\left(A^{2}+B^{2}\right)\right\}\right) \\
& =\min _{0 \leq t \leq 1}(\max \{(\tau-1) t, 1+(\tau-3) t\})=\frac{\tau-1}{2} .
\end{aligned}
$$

Combining (5.2) and (5.1), we arrive at

$$
2|\langle\boldsymbol{x}, \boldsymbol{w}\rangle|^{2}+1+(\tau-3)\left\langle|\boldsymbol{x}|^{2},|\boldsymbol{w}|^{2}\right\rangle \geq \min \left\{1, \frac{\tau-1}{2}\right\}
$$

for any $\boldsymbol{w}, \boldsymbol{x} \in \mathbb{R}^{n}$ such that $\|\boldsymbol{w}\|_{2}=\|\boldsymbol{x}\|_{2}=1$.

\subsection{Proof of Theorem 3.1 .}

Proof of Theorem 3.1. Since $\boldsymbol{W}_{l}=\boldsymbol{Z}_{l}-\frac{\eta_{l}}{m} \mathscr{P}_{T_{l}}\left(\mathscr{A}_{l}^{\top} \mathscr{A}_{l}\left(\boldsymbol{Z}_{l}\right)-\boldsymbol{y}\right)=\boldsymbol{Z}_{l}-\frac{\eta_{l}}{m} \mathscr{P}_{T_{l}} \mathscr{A}_{l}^{\top} \mathscr{A}_{l}\left(\boldsymbol{Z}_{l}-\boldsymbol{X}\right)$. we have

$$
\begin{aligned}
\left\|\boldsymbol{W}_{l}-\boldsymbol{X}\right\|_{F}= & \left\|\left(\boldsymbol{Z}_{l}-\boldsymbol{X}\right)-\frac{\eta_{l}}{m} \mathscr{P}_{T_{l}} \mathscr{A}_{l}^{\top} \mathscr{A}_{l}\left(\boldsymbol{Z}_{l}-\boldsymbol{X}\right)\right\|_{F} \\
\leq & \left\|\left(\mathscr{P}_{T_{l}}-\frac{\eta_{l}}{m} \mathscr{P}_{T_{l}} \mathscr{A}_{l}^{\top} \mathscr{A}_{l} \mathscr{P}_{T_{l}}\right)\left(\boldsymbol{Z}_{l}-\boldsymbol{X}\right)\right\|_{F}+\left\|\left(\boldsymbol{I}-\mathscr{P}_{T_{l}}\right) \boldsymbol{X}\right\|_{F} \\
& +\frac{\eta_{l}}{m}\left\|\mathscr{P}_{T_{z}} \mathscr{A}_{z}^{\top} \mathscr{A}_{z}\left(\boldsymbol{I}-\mathscr{P}_{T_{z}}\right)\left(\boldsymbol{Z}_{l}-\boldsymbol{X}\right)\right\|_{F} \cdot
\end{aligned}
$$

According to $\operatorname{TL}-\operatorname{RIP}(\alpha, \beta)$ and $\operatorname{TL}-\mathrm{WCP}(\theta)$, we have

$$
\left\|\left(\mathscr{P}_{T_{l}}-\frac{\alpha_{l}}{m} \mathscr{P}_{T_{l}} \mathscr{A}_{l}^{\top} \mathscr{A}_{l} \mathscr{P}_{T_{l}}\right)\left(\boldsymbol{Z}_{l}-\boldsymbol{X}\right)\right\|_{F} \leq \max \left\{\left|1-\eta_{l} \alpha\right|,\left|1-\eta_{l} \beta\right|\right\}\left\|\boldsymbol{Z}_{l}-\boldsymbol{X}\right\|_{F}
$$

and

$$
\frac{\eta_{l}}{m}\left\|\mathscr{P}_{T_{z}} \mathscr{A}_{z}^{\top} \mathscr{A}_{z}\left(\boldsymbol{I}-\mathscr{P}_{T_{z}}\right)\left(\boldsymbol{Z}_{l}-\boldsymbol{X}\right)\right\|_{F} \leq \eta_{l} \boldsymbol{\theta}\left\|\boldsymbol{Z}_{l}-\boldsymbol{X}\right\|_{F} .
$$

Applying (5.4), (5.5), and Lemma 4.1 in [7], inequality (5.3) becomes

$$
\left\|\boldsymbol{W}_{l}-\boldsymbol{X}\right\|_{F} \leq\left(\max \left\{\left|1-\eta_{l} \alpha\right|,\left|1-\eta_{l} \beta\right|\right\}+\eta_{l} \theta+\varepsilon_{0}\right)\left\|\boldsymbol{Z}_{l}-\boldsymbol{X}\right\|_{F}:=\mu\left\|\boldsymbol{Z}_{l}-\boldsymbol{X}\right\|_{F},
$$

where $\mu=\max \left\{\left|1-\eta_{l} \alpha\right|,\left|1-\eta_{l} \beta\right|\right\}+\eta_{l} \theta+\varepsilon_{0}$. According to Lemma 4.4 in [6], we have

$$
\left\|Z_{l+1}-\boldsymbol{X}\right\|_{F} \leq \mu \sqrt{1+16 \mu^{2} \varepsilon_{0}^{2}}\left\|Z_{l}-\boldsymbol{X}\right\|_{F}
$$

Denote $v:=\mu \sqrt{1+16 \mu^{2} \varepsilon_{0}^{2}}$. In order to obtain $v<1$, we should have $\mu \leq 1 / \sqrt{1+16 \varepsilon_{0}^{2}}$, which leads to

i.e.,

$$
\max \left\{\left|1-\eta_{l} \alpha\right|,\left|1-\eta_{l} \beta\right|\right\}+\eta_{l} \theta \leq 1 / \sqrt{1+16 \varepsilon_{0}^{2}}-\varepsilon_{0}
$$

$$
\eta_{l} \in\left[\frac{1-1 / \sqrt{1+16 \varepsilon_{0}^{2}}+\varepsilon_{0}}{\alpha-\theta}, \frac{1+1 / \sqrt{1+16 \varepsilon_{0}^{2}}-\varepsilon_{0}}{\beta+\theta}\right]
$$

provided that

$$
\theta<\alpha \text { and }\left(1-1 / \sqrt{1+16 \varepsilon_{0}^{2}}+\varepsilon_{0}\right) \beta+2 \theta \leq\left(1+1 / \sqrt{1+16 \varepsilon_{0}^{2}}-\varepsilon_{0}\right) \alpha
$$




\subsection{Proof of Theorem 3.2.}

Proof of Theorem 3.2. Based on Lemma 6.1, we have

$$
\begin{aligned}
& \boldsymbol{Y}_{R \sqrt{1-\delta}}:=\frac{1}{m} \sum_{j=1}^{m}\left|\left\langle\boldsymbol{x}, \boldsymbol{a}_{j}\right\rangle\right|^{2} \boldsymbol{a}_{j} \boldsymbol{a}_{j}^{\top} \mathbf{1}_{\left\{\left|\left\langle\boldsymbol{a}_{j}, \boldsymbol{x}\right\rangle\right| \leq R \sqrt{1-\delta}\|\boldsymbol{x}\|_{2}\right\}} \preceq \overline{\boldsymbol{Y}}_{R} \\
& \preceq \boldsymbol{Y}_{R \sqrt{1+\delta}}:=\frac{1}{m} \sum_{j=1}^{m}\left|\left\langle\boldsymbol{x}, \boldsymbol{a}_{j}\right\rangle\right|^{2} \boldsymbol{a}_{j} \boldsymbol{a}_{j}^{\top} \mathbf{1}_{\left\{\left|\left\langle\boldsymbol{a}_{j}, \boldsymbol{x}\right\rangle\right| \leq R \sqrt{1+\boldsymbol{\delta}}\|\boldsymbol{x}\|_{2}\right\}}
\end{aligned}
$$

with probability at least $1-\exp (-\underline{c} n)$ provided that $m \gtrsim \frac{K^{4}}{\delta^{2}} n$. Meanwhile, according to Lemma 6.3 , we have

$$
\left\|\overline{\boldsymbol{M}}_{R}-2 \boldsymbol{x} \boldsymbol{x}^{\top}-\right\| \boldsymbol{x}\left\|_{2}^{2} \boldsymbol{I}\right\| \leq\left(\boldsymbol{\delta} / 2+\left(1+\frac{|\tau-3|}{|\tau-1|}\right) 64 C^{4} K^{4} \exp \left(1-c_{0} R^{2}(1-\delta) /(C K)^{2}\right)\right)\|\boldsymbol{x}\|_{2}^{2} .
$$

with probability at least $1-\exp (-\underline{c} n)$ provided that

$$
m \geq \max \left\{\left(\frac{8 \bar{C} C^{2} R^{2}(1+\delta) K^{2}}{\left(1+\frac{|\tau-3|}{|\tau-1|}\right) \delta}\right)^{2} n,\left(\frac{8 \bar{C} C^{2} K^{2}}{\left(1+\frac{|\tau-3|}{|\tau-1|}\right) \delta}\right)^{2} n\right\}
$$

Take $R$ as an constant sufficiently large enough such that

$$
\delta / 2+\left(1+\frac{|\tau-3|}{|\tau-1|}\right) 64 C^{4} K^{4} \exp \left(1-c_{0} R(1-\delta) /(C K)^{2}\right) \leq \delta .
$$

Since the leading eigenvector of $\overline{\boldsymbol{M}}_{R}$ is $\boldsymbol{u}_{1}$, and $z_{0}=\sqrt{\frac{1}{m} \sum_{j=1}^{m} y_{i}} \boldsymbol{u}_{1}$, by standard calculation in [21], we have

$$
\min \left\{\left\|z_{0}-x\right\|_{2},\left\|z_{0}+x\right\|_{2}\right\} \leq \sqrt{5 \delta}\|x\|_{2} .
$$

Without loss of generality, assume that $\left\|z_{0}-x\right\|_{2} \leq \sqrt{5 \delta}\|x\|_{2}$. Here $\left\|z_{0}+x\right\|_{2} \leq \sqrt{5 \delta}\|x\|_{2}$ can be discussed similarly. Thus

$$
\left\|\boldsymbol{Z}_{0}-\boldsymbol{X}\right\|_{F} \leq\left\|\boldsymbol{z}_{0} \boldsymbol{z}_{0}^{\top}-\boldsymbol{x} \boldsymbol{x}^{\top}\right\|_{F} \leq\left\|\boldsymbol{z}_{0}\left(\boldsymbol{z}_{0}-\boldsymbol{x}\right)^{\top}\right\|_{F}+\left\|\left(\boldsymbol{z}_{0}-\boldsymbol{x}\right) \boldsymbol{x}^{\top}\right\|_{F} \leq(\sqrt{5 \delta}+2) \sqrt{5 \delta}\|\boldsymbol{X}\|_{F} .
$$

\subsection{Proof of Lemma 3.1.}

Proof of Lemma 3.1. First of all, we find that

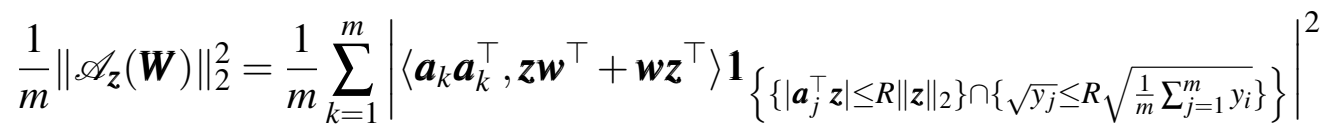

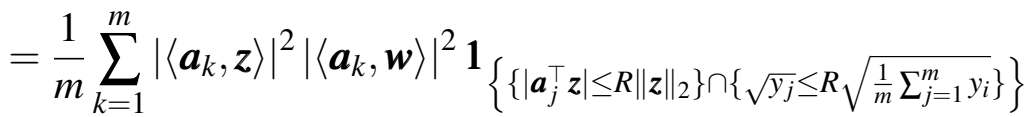

$$
\begin{aligned}
& =\boldsymbol{w}^{\top}\left(\frac{1}{m} \sum_{k=1}^{m}\left|\boldsymbol{a}_{k}^{\top} \boldsymbol{z}\right|^{2} \boldsymbol{a}_{k} \boldsymbol{a}_{k}^{\top} \mathbf{1}_{\left\{\left\{\left|\boldsymbol{a}_{j}^{\top} z\right| \leq R\|z\|_{2}\right\} \cap\left\{\sqrt{y_{j}} \leq R \sqrt{\frac{1}{m} \sum_{j=1}^{m} y_{i}}\right\}\right.}\right) \boldsymbol{w} \text {. }
\end{aligned}
$$


Applying Lemma 6.5, we have

$$
\begin{aligned}
& \mid \boldsymbol{w}^{\top}\left(\frac{1}{m} \sum_{k=1}^{m}\left|\boldsymbol{a}_{k}^{\top} \boldsymbol{z}\right|^{2} \boldsymbol{a}_{k} \boldsymbol{a}_{k}^{\top} \mathbf{1}_{\left\{\left\{\left|\boldsymbol{a}_{j}^{\top} z\right| \leq R\|z\|_{2}\right\} \cap\left\{\sqrt{y_{j}} \leq R \sqrt{\frac{1}{m} \sum_{j=1}^{m} y_{i}}\right\}\right)}\right) \boldsymbol{w} \\
& -\left(2 \boldsymbol{w}^{\top} \boldsymbol{z} \boldsymbol{z}^{\top} \boldsymbol{w}+\|\boldsymbol{z}\|_{2}^{2}\|\boldsymbol{w}\|_{2}^{2}+(\tau-3) \boldsymbol{w}^{\top} \operatorname{Diag}\left(|\boldsymbol{z}|^{2}\right) \boldsymbol{w}\right) \mid \\
& \leq\left(\left(5+4 R+6 R^{2} K^{2}\right) \boldsymbol{\varepsilon}+\left(16 K^{2} R^{4}+64 C^{4} K^{4}\right) \exp \left(1-\frac{c R^{2}}{K^{2}}\right)\right. \\
& \left.\quad+\frac{10 R^{2} K^{2}}{\varepsilon^{2}} \exp \left(1-\frac{c_{1}}{K^{2} \varepsilon^{2}}\right)\right)\|z\|_{2}^{2}\|\boldsymbol{w}\|_{2}^{2} .
\end{aligned}
$$

According to Lemma 5.1, we have

$$
\begin{aligned}
\min \left\{1, \frac{\tau-1}{2}\right\}\|z\|_{2}^{2}\|\boldsymbol{w}\|_{2}^{2} & \leq 2 \boldsymbol{w}^{\top} z z^{\top} \boldsymbol{w}+\|z\|_{2}^{2}\|\boldsymbol{w}\|_{2}^{2}+(\tau-3) \boldsymbol{w}^{\top} \operatorname{Diag}\left(|z|^{2}\right) \boldsymbol{w} \\
& \leq(3+|\tau-3|)\|\boldsymbol{z}\|_{2}^{2}\|\boldsymbol{w}\|_{2}^{2} .
\end{aligned}
$$

Taking $R^{2}=\frac{1}{\sqrt{\varepsilon}}$ and choosing $\varepsilon$ as a constant sufficiently small enough, we have

$$
\min \left\{\frac{1}{2}, \frac{\tau-1}{4}\right\}\|z\|_{2}^{2}\|\boldsymbol{w}\|_{2}^{2} \leq \frac{1}{m}\left\|\mathscr{A}_{Z}(\boldsymbol{W})\right\|_{2}^{2} \leq(3+2|\tau-3|)\|z\|_{2}^{2}\|\boldsymbol{w}\|_{2}^{2} \text {. }
$$

Since $\boldsymbol{W}=\boldsymbol{z} \boldsymbol{w}^{\top}+\boldsymbol{w} \boldsymbol{z}^{\top}$, we have $\|\boldsymbol{W}\|_{F}^{2}=2\|\boldsymbol{z}\|_{2}^{2}\|\boldsymbol{w}\|_{2}^{2}+2\left|z^{\top} \boldsymbol{w}\right|^{2} \leq 4\|z\|_{2}^{2}\|\boldsymbol{w}\|_{2}^{2}$. Therefore,

$$
\min \left\{\frac{1}{8}, \frac{\tau-1}{16}\right\}\|\boldsymbol{W}\|_{F}^{2} \leq \frac{1}{m}\left\|\mathscr{A}_{Z}(\boldsymbol{W})\right\|_{2}^{2} \leq \frac{3+2|\tau-3|}{2}\|\boldsymbol{W}\|_{F}^{2}
$$

\subsection{Proof of Lemma 3.2.}

Proof of Lemma 3.2. Withou loss of generality, assume that $\|\boldsymbol{x}\|_{2}=1$. Applying Lemma 3.1, we have

$$
\left\|\frac{1}{\sqrt{m}} \mathscr{P}_{T_{z}} \mathscr{A}_{z}^{\top}\right\| \leq \sqrt{\frac{3+2|\tau-3|}{2}} .
$$

The only thing we need to do is to estimate the upper bound of $\frac{1}{\sqrt{m}}\left\|\mathscr{A}_{z}\left(\boldsymbol{I}-\mathscr{P}_{T_{z}}\right)\left(\boldsymbol{z} \boldsymbol{z}^{\top}-\boldsymbol{x} \boldsymbol{x}^{\top}\right)\right\|_{2}$. Letting $\boldsymbol{h}=\boldsymbol{z}-\boldsymbol{x}$, we have

$$
\left(\boldsymbol{I}-\mathscr{P}_{T_{z}}\right)\left(\boldsymbol{z} \boldsymbol{z}^{\top}-\boldsymbol{x} \boldsymbol{x}^{\top}\right)=-\left(\frac{\boldsymbol{z}^{\top} \boldsymbol{h}}{\|\boldsymbol{z}\|_{2}^{2}} \boldsymbol{z}-\boldsymbol{h}\right)\left(\frac{\boldsymbol{z}^{\top} \boldsymbol{h}}{\|\boldsymbol{z}\|_{2}^{2}} \boldsymbol{z}-\boldsymbol{h}\right)^{\top} .
$$

If $\left|\boldsymbol{a}_{k}^{\top} z\right| \leq R\|z\|_{2}$ and $\left|\boldsymbol{a}_{k}^{\top} \boldsymbol{x}\right| \leq R\|\boldsymbol{x}\|_{2}$, then

$$
\left|\boldsymbol{a}_{k}^{\top} \boldsymbol{h}\right| \leq\left|\boldsymbol{a}_{k}^{\top} \boldsymbol{z}\right|+\left|\boldsymbol{a}_{k}^{\top} \boldsymbol{x}\right| \leq R\left(\|\boldsymbol{z}\|_{2}+\|\boldsymbol{x}\|_{2}\right) \leq R\left(2+\frac{\varepsilon_{0}}{1-\sqrt{5 / 4} \varepsilon_{0}}\right)\|z\|_{2} \leq 3 R\|z\|_{2} .
$$


The third inequality is according to $\left\|\boldsymbol{z} \boldsymbol{z}^{\top}-\boldsymbol{x} \boldsymbol{x}^{\top}\right\|_{F}^{2} \geq \frac{4}{5}\|\boldsymbol{z}-\boldsymbol{x}\|_{2}^{2}\|\boldsymbol{x}\|_{2}^{2}$ in [6, Lemma 5.5], which leads to $\|\boldsymbol{z}-\boldsymbol{x}\|_{2} \leq \sqrt{\frac{5}{4}} \varepsilon_{0}$, and $\|\boldsymbol{z}\|_{2} \geq 1-\sqrt{\frac{5}{4}} \varepsilon_{0}$. The last inequality is based on $\varepsilon \leq \frac{1}{2}$. Therefore,

$$
\begin{aligned}
& \frac{1}{m}\left\|\mathscr{A}_{z}\left(\boldsymbol{I}-\mathscr{P}_{T_{z}}\right)\left(z z^{\top}-\boldsymbol{x} \boldsymbol{x}^{\top}\right)\right\|_{2}^{2}
\end{aligned}
$$

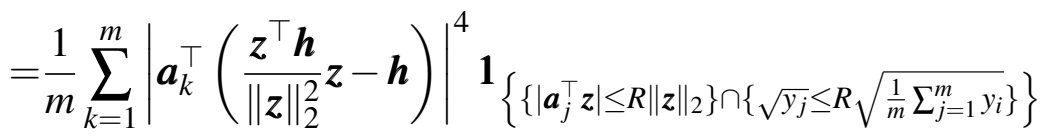

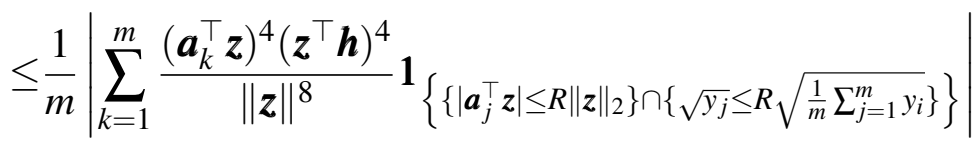

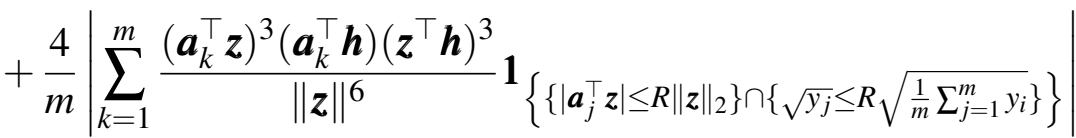

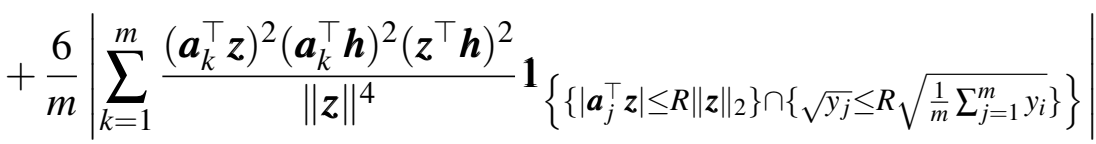

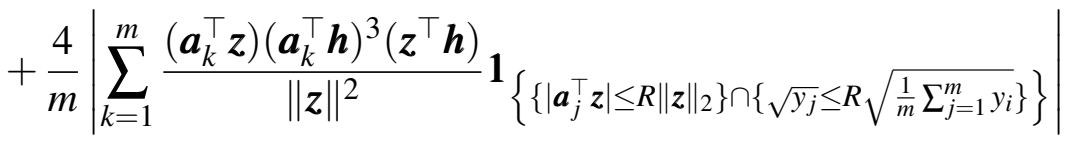

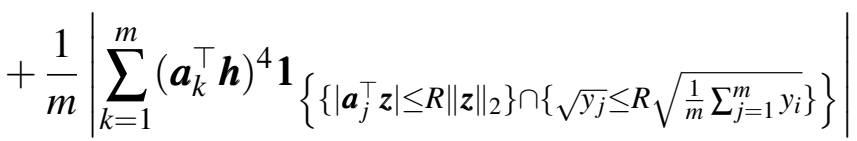

$$
\begin{aligned}
& \leq 2 R^{4}\|\boldsymbol{h}\|_{2}^{4}+5 R^{3}\|\boldsymbol{h}\|_{2}^{4}+8 R^{2}\|\boldsymbol{h}\|_{2}^{4}+16 R\|\boldsymbol{h}\|_{2}^{3}\|\boldsymbol{z}\|_{2}+5 R^{4} K^{2} \exp \left(1-\frac{c_{1} R^{2}}{K^{2}}\right)\|\boldsymbol{h}\|_{2}^{2}\|\boldsymbol{z}\|_{2}^{2} \\
& \leq\left(\left(2 R^{4}+5 R^{3}+8 R^{2}\right) \frac{\|\boldsymbol{h}\|_{2}^{2}}{\|\boldsymbol{z}\|_{2}^{2}}+16 R \frac{\|\boldsymbol{h}\|_{2}}{\|\boldsymbol{z}\|_{2}}+5 R^{4} K^{2} \exp \left(1-\frac{c_{1} R^{2}}{K^{2}}\right)\right)\|\boldsymbol{h}\|_{2}^{2}\|\boldsymbol{z}\|_{2}^{2} .
\end{aligned}
$$

The second inequality is based on (5.6), Lemma 6.1, and Lemma 6.4. If condition (3.2) holds, then

$$
\frac{\|\boldsymbol{h}\|_{2}}{\|\boldsymbol{x}\|_{2}} \leq \sqrt{5 / 4} \frac{\left\|z z^{\top}-\boldsymbol{x} \boldsymbol{x}^{T}\right\|_{F}}{\|\boldsymbol{X}\|_{F}} \leq 2 \varepsilon_{0} \leq \min \left\{\sqrt{\frac{\delta}{2\left(2 R^{4}+5 R^{3}+8 R^{2}\right)}}, \frac{\delta}{32 R}, \frac{1}{2}\right\} .
$$

Substituting (5.9) into (5.8), we can obtain the conclusion immediately.

\section{APPENDIX}

6.1. Properties of sub-Gaussian random variables. Here we show some properties for subGaussian random vectors. A random vector $\boldsymbol{a} \in \mathbb{R}^{n}$ is sub-Gaussian if the one-dimensional marginals $\langle\boldsymbol{a}, \boldsymbol{u}\rangle$ are sub-Gaussian random variables for all $\boldsymbol{u} \in \mathbb{R}^{n}$. The sub-Gaussian norm of $a$ is defined as

$$
\|\boldsymbol{a}\|_{\psi_{2}}=\sup _{\boldsymbol{u} \in \mathbb{S}^{n-1}}\|\langle\boldsymbol{a}, \boldsymbol{u}\rangle\|_{\psi_{2}} .
$$

Theorem 6.1. [5, Lemma 5.24] Let $x_{1}, \ldots, x_{n}$ be independent centered sub-Gaussian random variables. Then $\boldsymbol{x}=\left[x_{1}, \ldots, x_{n}\right]^{\top}$ is a centered sub-Gaussian random vector in $\mathbb{R}^{n}$, and

$$
\|\boldsymbol{x}\|_{\psi_{2}} \leq C \max _{i \leq n}\left\|x_{i}\right\|_{\psi_{2}}
$$


where $C$ is an positive absolute constant.

Theorem 6.2. [5, Remark 5.40] Suppose that $\boldsymbol{a}_{i} \in \mathbb{R}^{n}(i=1, \ldots, m)$ are independently copies of sub-Gaussian random vector $\boldsymbol{a} \in \mathbb{R}^{n}$ with $\mathbb{E} \boldsymbol{a} \boldsymbol{a}^{\top}=\boldsymbol{\Sigma}$, and $\|\boldsymbol{a}\|_{\psi_{2}} \leq C_{\psi_{2}}$. Then, for every $t \geq 0$, the following inequality holds with probability at least $1-2 \exp \left(-\underline{\underline{c}} t^{2} / C_{\psi_{2}}^{4}\right)$,

$$
\left\|\frac{1}{m} \sum_{i=1}^{m} \boldsymbol{a}_{i} \boldsymbol{a}_{i}^{\top}-\boldsymbol{\Sigma}\right\| \leq \max \left\{\boldsymbol{\xi}, \xi^{2}\right\},
$$

where $\xi=\bar{C} C_{\psi_{2}}^{2} \sqrt{\frac{n}{m}}+\frac{t}{\sqrt{m}}$, and $\bar{C}$ and $\underline{c}$ are two positive absolute constants.

6.2. Technical tools in the proof of Theorem 3.2. The elements of $\boldsymbol{A} \in \mathbb{R}^{m \times n}$ are independently copies of sub-Gaussian random variable $a$ in Definition 1.2 with parameters $K$ and $\tau$. $\boldsymbol{a}_{j}^{\top}$ is the $j$-th row of $\boldsymbol{A}$. First of all, we show some important concentration inequalities on $\boldsymbol{A}$.

Lemma 6.1. With probability at least $1-2 \exp (-\underline{c} n)$, we have

$$
\left\|\frac{1}{m} \sum_{j=1}^{m} \boldsymbol{a}_{j} \boldsymbol{a}_{j}^{\top}-\boldsymbol{I}\right\| \leq \boldsymbol{\delta},
$$

provided $m \gtrsim \frac{K^{4}}{\delta^{2}} n$. Here $\delta<1$ is some positive constant, and $\underline{\mathrm{c}}$ is some absolute constant.

Proof. Applying Theorem 6.1, we have $\left\|\boldsymbol{a}_{j}\right\|_{\psi_{2}} \leq C K$, for $j=1, \ldots, m$. Taking

$$
C_{\psi_{2}}=C K, m \geq\left(\frac{2 \bar{C} C^{2} K^{2}}{\delta}\right)^{2} n, \text { and } t=\bar{C} C^{2} K^{2} \sqrt{n}
$$

in Theorem 6.2, we have $\xi \leq \delta$, and

$$
\left\|\frac{1}{m} \sum_{j=1}^{m} \boldsymbol{a}_{j} \boldsymbol{a}_{j}^{\top}-\boldsymbol{I}\right\| \leq \boldsymbol{\delta}
$$

with probability at least $1-\exp (-\underline{c} n)$.

Lemma 6.2. For any fixed $\boldsymbol{x} \in \mathbb{R}^{n}$ and $\delta<1$, denote

$$
\boldsymbol{Y}_{R}:=\frac{1}{m} \sum_{j=1}^{m}\left|\left\langle\boldsymbol{x}, \boldsymbol{a}_{j}\right\rangle\right|^{2} \boldsymbol{a}_{j} \boldsymbol{a}_{j}^{\top} \mathbf{1}_{\left\{\left|\left\langle\boldsymbol{a}_{j}, \boldsymbol{x}\right\rangle\right| \leq R\|\boldsymbol{x}\|_{2}\right\}} .
$$

With probability at least $1-2 \exp (-\underline{c} n)$, we have

$$
\begin{aligned}
& \left\|\boldsymbol{Y}_{R}-\left(2 \boldsymbol{x} \boldsymbol{x}^{\top}+\|\boldsymbol{x}\|_{2}^{2} \boldsymbol{I}+\left.(\tau-3) \operatorname{Diag}(\mid \boldsymbol{x})\right|^{2}\right)\right\| \\
\leq & \left(\frac{\delta}{2}+64 C^{4} K^{4} \exp \left(1-c_{0} R^{2} /(C K)^{2}\right)\right)\|\boldsymbol{x}\|_{2}^{2},
\end{aligned}
$$

provided $m \geq C_{\delta, R, K} n$. Here $C, \underline{c}$ and $c_{0}$ are absolute constants.

Proof. Without loss of generality, assume that $\|\boldsymbol{x}\|_{2}=1$. Then $\boldsymbol{Y}_{R}$ becomes

$$
\boldsymbol{Y}_{R}=\frac{1}{m} \sum_{j=1}^{m}\left|\left\langle\boldsymbol{x}, \boldsymbol{a}_{j}\right\rangle\right|^{2} \boldsymbol{a}_{j} \boldsymbol{a}_{j}^{\top} \mathbf{1}_{\left\{\left|\left\langle\boldsymbol{a}_{j}, \boldsymbol{x}\right\rangle\right| \leq R\right\}},
$$


and it is equivalent to estimate the upper bound of $\left\|\boldsymbol{Y}_{R}-2 \boldsymbol{x} \boldsymbol{x}^{\top}-\boldsymbol{I}-(\tau-3) \operatorname{Diag}\left(|\boldsymbol{x}|^{2}\right)\right\|$. Since

$$
\begin{aligned}
& \left\|\boldsymbol{Y}_{R}-2 \boldsymbol{x} \boldsymbol{x}^{\top}-\boldsymbol{I}-(\tau-3) \operatorname{Diag}\left(|\boldsymbol{x}|^{2}\right)\right\| \\
= & \left\|\boldsymbol{Y}_{R}-\mathbb{E} \boldsymbol{Y}_{R}+\mathbb{E} \boldsymbol{Y}_{R}-2 \boldsymbol{x} \boldsymbol{x}^{\top}-\boldsymbol{I}-(\tau-3) \operatorname{Diag}\left(|\boldsymbol{x}|^{2}\right)\right\| \\
\leq & \left\|\boldsymbol{Y}_{R}-\mathbb{E} \boldsymbol{Y}_{R}\right\|+\left\|\mathbb{E} \boldsymbol{Y}_{R}-2 \boldsymbol{x} \boldsymbol{x}^{\top}-\boldsymbol{I}-(\tau-3) \operatorname{Diag}\left(|\boldsymbol{x}|^{2}\right)\right\|,
\end{aligned}
$$

we need to estimate $\left\|\boldsymbol{Y}_{R}-\mathbb{E} \boldsymbol{Y}_{R}\right\|$ and $\left\|\mathbb{E} \boldsymbol{Y}_{R}-2 \boldsymbol{x} \boldsymbol{x}^{\top}-\boldsymbol{I}-(\tau-3) \operatorname{Diag}\left(|\boldsymbol{x}|^{2}\right)\right\|$ separatively.

(a) Estimation of $\left\|Y_{R}-\mathbb{E} \boldsymbol{Y}_{R}\right\|$.

Denote $\boldsymbol{b}_{j}=\left|\left\langle\boldsymbol{x}, \boldsymbol{a}_{j}\right\rangle\right| \boldsymbol{a}_{j} \mathbf{1}_{\left\{\left\langle\boldsymbol{a}_{j}, \boldsymbol{x}\right\rangle \mid \leq R\right\}}$. We have $\boldsymbol{Y}_{R}=\frac{1}{m} \sum_{j=1}^{m} \boldsymbol{b}_{j} \boldsymbol{b}_{j}^{\top}$. For any $\boldsymbol{u} \in \mathbb{S}^{n-1}$ and any integer $p \geq 1$, we have

$$
\left|\left\langle\boldsymbol{b}_{j}, \boldsymbol{u}\right\rangle\right|^{p}=\left|\left\langle\boldsymbol{x}, \boldsymbol{a}_{j}\right\rangle\right|^{p}\left|\left\langle\boldsymbol{a}_{j}, \boldsymbol{u}\right\rangle\right|^{p} \mathbf{1}_{\left\{\left\langle\boldsymbol{a}_{j}, \boldsymbol{x}\right\rangle \mid \leq R\right\}} \leq R^{p}\left|\left\langle\boldsymbol{a}_{j}, \boldsymbol{u}\right\rangle\right|^{p} .
$$

Therefore, according to Theorem 6.1, we have $\left\|\boldsymbol{b}_{j}\right\|_{\psi_{2}} \leq R\left\|\boldsymbol{a}_{j}\right\|_{\psi_{2}} \leq C R K$. In Theorem 6.2, taking

$$
\boldsymbol{\Sigma}=\mathbb{E} \boldsymbol{b}_{1} \boldsymbol{b}_{1}^{\top}, C_{\psi_{2}}=C R K, m \geq\left(\frac{4 \bar{C} C^{2} R^{2} K^{2}}{\delta}\right)^{2} n, \text { and } t=\bar{C} C^{2} R^{2} K^{2} \sqrt{n}
$$

we have

$$
\left\|\boldsymbol{Y}_{R}-\mathbb{E} \boldsymbol{Y}_{R}\right\|=\left\|\boldsymbol{Y}_{R}-\boldsymbol{\Sigma}\right\| \leq \frac{\delta}{2}
$$

with probability at least $1-2 \exp (-\underline{c} n)$.

(b) Estimation of $\left\|\mathbb{E} \boldsymbol{Y}_{R}-2 \boldsymbol{x} \boldsymbol{x}^{\top}-\boldsymbol{I}-(\tau-3) \operatorname{Diag}\left(|\boldsymbol{x}|^{2}\right)\right\|$.

For any fixed $\boldsymbol{u} \in \mathbb{S}^{n-1}$, we have

$$
\begin{aligned}
& \boldsymbol{u}^{\top}\left(\mathbb{E} \boldsymbol{Y}_{R}-2 \boldsymbol{x} \boldsymbol{x}^{\top}-\boldsymbol{I}-(\tau-3) \operatorname{Diag}\left(|\boldsymbol{x}|^{2}\right)\right) \boldsymbol{u} \\
= & \boldsymbol{u}^{\top} \mathbb{E}\left(\left|\left\langle\boldsymbol{a}_{1}, \boldsymbol{x}\right\rangle\right|^{2} \boldsymbol{a}_{1} \boldsymbol{a}_{1}^{\top} \mathbf{1}_{\left\{\left\langle\boldsymbol{a}_{\mathbf{1}}, \boldsymbol{x}\right\rangle \mid \leq \mathbf{R}\right\}}-\left|\left\langle\boldsymbol{a}_{1}, \boldsymbol{x}\right\rangle\right|^{2} \boldsymbol{a}_{1} \boldsymbol{a}_{1}^{\top}\right) \boldsymbol{u}=\mathbb{E}\left(\left|\left\langle\boldsymbol{a}_{1}, \boldsymbol{x}\right\rangle\right|^{2}\left|\left\langle\boldsymbol{a}_{1}, \boldsymbol{u}\right\rangle\right|^{2} \mathbf{1}_{\left\{\left\langle\boldsymbol{a}_{\mathbf{1}}, \boldsymbol{x}\right\rangle \mid>\mathbf{R}\right\}}\right) \\
\leq & \left(\mathbb{E}\left(\left|\left\langle\boldsymbol{a}_{1}, \boldsymbol{x}\right\rangle\right|^{4}\left|\left\langle\boldsymbol{a}_{1}, \boldsymbol{u}\right\rangle\right|^{4}\right)\right)^{1 / 2}\left(\mathbb{P}\left(\left\langle\boldsymbol{a}_{1}, \boldsymbol{x}\right\rangle \mid>R\right)\right)^{1 / 2} \\
\leq & \left(\mathbb{E}\left|\left\langle\boldsymbol{a}_{1}, \boldsymbol{x}\right\rangle\right|^{8}\right)^{1 / 4}\left(\mathbb{E}\left|\left\langle\boldsymbol{a}_{1}, \boldsymbol{u}\right\rangle\right|^{8}\right)^{1 / 4}\left(\mathbb{P}\left(\left\langle\boldsymbol{a}_{1}, \boldsymbol{x}\right\rangle \mid>R\right)\right)^{1 / 2} \\
\leq & (\sqrt{8} C K)^{4} \exp \left(1-c_{0} R^{2} /(C K)^{2}\right)=64 C^{4} K^{4} \exp \left(1-c_{0} R^{2} /(C K)^{2}\right) .
\end{aligned}
$$

The third line is according to Cauchy-Swartcz inequality and the definition of sub-Gaussian vector norm. The fifth line is based on (1.2). It leads to

$$
\left\|\mathbb{E} \boldsymbol{Y}_{R}-2 \boldsymbol{x} \boldsymbol{x}^{\top}-\boldsymbol{I}-(\tau-3) \operatorname{Diag}\left(|\boldsymbol{x}|^{2}\right)\right\| \leq 64 C^{4} K^{4} \exp \left(1-c_{0} R^{2} /(C K)^{2}\right) .
$$

Combining (6.2) and (6.5), we can obtain that

$$
\left\|\boldsymbol{Y}_{R}-2 \boldsymbol{x} \boldsymbol{x}^{\top}-\boldsymbol{I}-(\tau-3) \operatorname{Diag}\left(|\boldsymbol{x}|^{2}\right)\right\| \leq \frac{\delta}{2}+64 C^{4} K^{4} \exp \left(1-c_{0} R^{2} /(C K)^{2}\right)
$$

with probability at least $1-2 \exp (-\underline{c} n)$.

Lemma 6.3. For any fixed $\boldsymbol{x} \in \mathbb{R}^{n}$ and $\delta<1$, denote $\boldsymbol{Y}_{R}$ as in (6.1) and

$$
\boldsymbol{M}_{R}:=\boldsymbol{Y}_{R}-\frac{\tau-3}{\tau-1} \operatorname{Diag}\left(\boldsymbol{Y}_{R}-\frac{1}{m} \sum_{j=1}^{m}\left|\left\langle\boldsymbol{a}_{j}, \boldsymbol{x}\right\rangle\right|^{2} \boldsymbol{I}\right) .
$$


Then with probability at least $1-2 \exp (-\underline{c} n)$, we have

$$
\left\|\boldsymbol{M}_{R}-2 \boldsymbol{x} \boldsymbol{x}^{\top}-\right\| \boldsymbol{x}\left\|_{2}^{2} \boldsymbol{I}\right\| \leq\left(\boldsymbol{\delta} / 2+\left(1+\frac{|\tau-3|}{|\tau-1|}\right) 64 C^{4} K^{4} \exp \left(1-c_{0} R^{2} /(C K)^{2}\right)\right)\|\boldsymbol{x}\|_{2}^{2}
$$

provided $m \geq C_{\delta, R, K, \tau} n$. Here $C_{\delta, R, K, \tau}$ can be seen in (6.7).

Proof. Assume that $\|\boldsymbol{x}\|_{2}=1$. We can find that

$$
\begin{aligned}
& \left\|\boldsymbol{M}_{R}-2 \boldsymbol{x} \boldsymbol{x}^{\top}-\right\| \boldsymbol{x}\left\|_{2}^{2} \boldsymbol{I}\right\|=\left\|\boldsymbol{M}_{R}-2 \boldsymbol{x} \boldsymbol{x}^{\top}-\boldsymbol{I}\right\| \\
= & \left\|\boldsymbol{M}_{R}-\mathbb{E} \boldsymbol{M}_{R}+\mathbb{E} \boldsymbol{M}_{R}-2 \boldsymbol{x} \boldsymbol{x}^{\top}-\right\| \boldsymbol{x}\left\|_{2}^{2} \boldsymbol{I}\right\| \\
\leq & \left\|\boldsymbol{M}_{R}-\mathbb{E} \boldsymbol{M}_{R}\right\|+\left\|\mathbb{E} \boldsymbol{M}_{R}-2 \boldsymbol{x} \boldsymbol{x}^{\top}-\right\| \boldsymbol{x}\left\|_{2}^{2} \boldsymbol{I}\right\| \\
\leq & \left\|\boldsymbol{Y}_{R}-\mathbb{E} \boldsymbol{Y}_{R}\right\|+\frac{\tau-3}{\tau-1}\left\|\operatorname{Diag}\left(\boldsymbol{Y}_{R}-\frac{1}{m} \sum_{j=1}^{m}\left|\left\langle\boldsymbol{a}_{j}, \boldsymbol{x}\right\rangle\right|^{2} \boldsymbol{I}\right)-\mathbb{E} \operatorname{Diag}\left(\boldsymbol{Y}_{R}-\frac{1}{m} \sum_{j=1}^{m}\left|\left\langle\boldsymbol{a}_{j}, \boldsymbol{x}\right\rangle\right|^{2} \boldsymbol{I}\right)\right\| \\
& +\left\|\mathbb{E} \boldsymbol{M}_{R}-2 \boldsymbol{x} \boldsymbol{x}^{\top}-\right\| \boldsymbol{x}\left\|_{2}^{2} \boldsymbol{I}\right\| \\
\leq & \left(1+\frac{|\tau-3|}{|\tau-1|}\right)\left\|\boldsymbol{Y}_{R}-\mathbb{E} \boldsymbol{Y}_{R}\right\|+\left.\frac{|\tau-3|}{|\tau-1|}\left|\frac{1}{m} \sum_{j=1}^{m}\right|\left\langle\boldsymbol{a}_{j}, \boldsymbol{x}\right\rangle\right|^{2}-\mathbb{E}\left(\frac{1}{m} \sum_{j=1}^{m}\left|\left\langle\boldsymbol{a}_{j}, \boldsymbol{x}\right\rangle\right|^{2}\right) \mid \\
& +\left\|\mathbb{E} \boldsymbol{M}_{R}-2 \boldsymbol{x} \boldsymbol{x}^{\top}-\right\| \boldsymbol{x}\left\|_{2}^{2} \boldsymbol{I}\right\| \\
\leq & \frac{\boldsymbol{\delta}}{4}+\frac{\boldsymbol{\delta}}{4}+\left\|\mathbb{E} \boldsymbol{M}_{R}-2 \boldsymbol{x} \boldsymbol{x}^{\top}-\right\| \boldsymbol{x}\left\|_{2}^{2} \boldsymbol{I}\right\|,
\end{aligned}
$$

provided

$$
m \geq \max \left\{\left(\frac{8 \bar{C} C^{2} R^{2} K^{2}}{\left(1+\frac{|\tau-3|}{|\tau-1|}\right) \delta}\right)^{2} n,\left(\frac{8 \bar{C} C^{2} K^{2}}{\left(1+\frac{|\tau-3|}{|\tau-1|}\right) \delta}\right)^{2} n\right\}
$$

The last line is according to inequality (6.2) and Lemma 6.1. In addition,

$$
\begin{aligned}
& \left\|\mathbb{E} \boldsymbol{M}_{R}-2 \boldsymbol{x} \boldsymbol{x}^{\top}-\right\| \boldsymbol{x}\left\|_{2}^{2} \boldsymbol{I}\right\| \\
= & \sup _{\boldsymbol{u} \in \mathbb{S}^{n-1}}\left|\boldsymbol{u}^{\top}\left(\mathbb{E}\left|\left\langle\boldsymbol{a}_{1}, \boldsymbol{x}\right\rangle\right|^{2} \boldsymbol{a}_{1} \boldsymbol{a}_{1}^{\top} \mathbf{1}_{\left\{\left\langle\boldsymbol{a}_{1}, \boldsymbol{x}\right\rangle \mid>\mathbf{R}\right\}}-\frac{\tau-3}{\tau-1} \mathbb{E D i a g}\left(\left|\left\langle\boldsymbol{a}_{1}, \boldsymbol{x}\right\rangle\right|^{2} \boldsymbol{a}_{1} \boldsymbol{a}_{1}^{\top} \mathbf{1}_{\left\{\left\langle\boldsymbol{a}_{\mathbf{1}}, \boldsymbol{x}\right\rangle \mid>\mathbf{R}\right.}\right)\right) \boldsymbol{u}\right| \\
\leq & \left.\left(1+\frac{|\tau-3|}{|\tau-1|}\right) \sup _{\boldsymbol{u} \in \mathbb{S}^{n-1}}\left|\boldsymbol{u}^{\top} \mathbb{E}\right|\left\langle\boldsymbol{a}_{1}, \boldsymbol{x}\right\rangle\right|^{2} \boldsymbol{a}_{1} \boldsymbol{a}_{1}^{\top} \mathbf{1}_{\left\{\left\langle\boldsymbol{a}_{\mathbf{1}}, \boldsymbol{x}\right\rangle \mid>\mathbf{R}\right\}} \boldsymbol{u} \mid \\
\leq & \left(1+\frac{|\tau-3|}{|\tau-1|}\right) 64 C^{4} K^{4} \exp \left(1-c_{0} R^{2} /(C K)^{2}\right)
\end{aligned}
$$

The last line is according to (6.4). Combining (6.6) and (6.8), we have

$$
\left\|\boldsymbol{M}_{R}-2 \boldsymbol{x} \boldsymbol{x}^{\top}-\right\| \boldsymbol{x}\left\|_{2}^{2} \boldsymbol{I}\right\| \leq \boldsymbol{\delta} / 2+\left(1+\frac{|\tau-3|}{|\tau-1|}\right) 64 C^{4} K^{4} \exp \left(1-c_{0} R^{2} /(C K)^{2}\right) .
$$


6.3. Technical tools in the proof of Theorem 3.3. The elements of $\boldsymbol{A} \in \mathbb{R}^{m \times n}$ are independently copies of sub-Gaussian random variable $a$ in Definition 1.2 with parameters $K$ and $\tau$. $\boldsymbol{a}_{j}^{\top}$ is the $j$-th row of $\boldsymbol{A}$. Lemma 5.3 in [6] can be applied to sub-Gaussian case by slightly modification, thus we omit the proof.

Lemma 6.4. Fix $R \geq 1$ and let $\varepsilon \in(0,1)$ be a sufficiently small constant. With probability at least $1-\exp \left(-c_{K} m \varepsilon^{2}\right)$,

$$
\left\|\frac{1}{m} \sum_{k=1}^{m} \boldsymbol{a}_{k} \boldsymbol{a}_{k}^{\top} \mathbf{1}_{\left\{\left|\boldsymbol{a}_{k}^{\top} z\right|>R\|z\|_{2}\right\}}\right\| \leq 5 K^{2} R^{2} \exp \left(1-\frac{c_{1} R^{2}}{K^{2}}\right)+\varepsilon
$$

holds uniformly for all $\|z\| \neq 0$ provided $m \geq C_{K} \varepsilon^{-2} \log \varepsilon^{-1} n$. Here $C_{K}$ and $c_{K}$ are constants depending on $K$.

The following lemma provides a uniform bound, which is different from Lemma 6.2.

Lemma 6.5. Fix $R \geq 2$ and let $\varepsilon \in(0,1)$ be a sufficiently small constant. With probability at least $1-\exp \left(-c_{K} m \varepsilon^{2}\right)$, we have

$$
\begin{aligned}
& \left\|\frac{1}{m} \sum_{k=1}^{m}\left|\boldsymbol{a}_{k}^{\top} \boldsymbol{z}\right|^{2} \boldsymbol{a}_{k} \boldsymbol{a}_{k}^{\top} \mathbf{1}_{\left\{\left\{\left|\boldsymbol{a}_{j}^{\top} \boldsymbol{z}\right| \leq R\|\boldsymbol{z}\|_{2}\right\}\right\}}-\left(2 \boldsymbol{z} \boldsymbol{z}^{\top}+\|\boldsymbol{z}\|_{2}^{2} \boldsymbol{I}+(\tau-3) \operatorname{Diag}\left(|\boldsymbol{z}|^{2}\right)\right)\right\| \\
\leq & \left(\left(5+4 R+4 R^{2}\right) \boldsymbol{\varepsilon}+\left(10 K^{2} R^{4}+64 C^{4} K^{4}\right) \exp \left(1-\frac{c R^{2}}{K^{2}}\right)+\frac{10 R^{2} K^{2}}{\varepsilon^{2}} \exp \left(1-\frac{c_{1}}{K^{2} \varepsilon^{2}}\right)\right)\|\boldsymbol{z}\|_{2}^{2}
\end{aligned}
$$

holds for all $z \in \mathbb{R}^{n}$ provided that $m \geq C_{K, R} \varepsilon^{-2} \log \varepsilon^{-1} n$. Besides,

$$
\begin{aligned}
& \left\|\frac{1}{m} \sum_{k=1}^{m}\left|\boldsymbol{a}_{k}^{\top} \boldsymbol{z}\right|^{2} \boldsymbol{a}_{k} \boldsymbol{a}_{k}^{\top} \mathbf{1}_{\left\{\left\{\left|\boldsymbol{a}_{j}^{\top} z\right| \leq R\|\boldsymbol{z}\|_{2}\right\} \cap\left\{\sqrt{y_{j}} \leq R \sqrt{\frac{1}{m} \sum_{j=1}^{m} y_{i}}\right\}\right.}-\left(2 \boldsymbol{z} \boldsymbol{z}^{\top}+\|\boldsymbol{z}\|_{2}^{2} \boldsymbol{I}+(\tau-3) \operatorname{Diag}\left(|\boldsymbol{z}|^{2}\right)\right)\right\| \\
& \leq\left(\left(5+4 R+6 R^{2} K^{2}\right) \varepsilon+\left(16 K^{2} R^{4}+64 C^{4} K^{4}\right) \exp \left(1-\frac{c R^{2}}{K^{2}}\right)+\frac{10 R^{2} K^{2}}{\varepsilon^{2}} \exp \left(1-\frac{c_{1}}{K^{2} \varepsilon^{2}}\right)\right)\|z\|_{2}^{2} .
\end{aligned}
$$

Proof. Set $\boldsymbol{Y}_{R, z}:=\frac{1}{m} \sum_{k=1}^{m}\left|\boldsymbol{a}_{k}^{\top} \boldsymbol{z}\right|^{2} \boldsymbol{a}_{k} \boldsymbol{a}_{k}^{\top} \mathbf{1}_{\left\{\left\{\left|\boldsymbol{a}_{j}^{\top} z\right| \leq R\|z\|_{2}\right\}\right\}}$. Applying Lemma 6.2, for any fixed $z \in \mathbb{S}^{n-1}$, we have

$$
\left\|\boldsymbol{Y}_{R, z}-2 z z^{\top}-\boldsymbol{I}-(\tau-3) \operatorname{Diag}\left(|z|^{2}\right)\right\| \leq \frac{1+R^{2} K^{2}}{2} \varepsilon+64 C^{4} K^{4} \exp \left(1-c_{0} R^{2} /(C K)^{2}\right)
$$

with probability at least $1-2 \exp \left(-c m \varepsilon^{2}\right)$ provided $m \gtrsim \frac{R^{4} K^{4}}{\varepsilon^{2}} n$. In order to show the uniform bound for all $z \in \mathbb{S}^{n-1}$, we first find that, for all $z \in \mathscr{N}_{\varepsilon^{2}}$, inequality (6.9) holds provided $m \gtrsim$ $\frac{R^{4} K^{4} \log \varepsilon^{-1}}{\varepsilon^{2}} n$. Here $\mathscr{N}_{\varepsilon^{2}}$ denotes a $\varepsilon^{2}$-net of $\mathbb{S}^{n-1}$, i.e., for any $z \in \mathbb{S}^{n-1}$, there exists $z_{0} \in \mathscr{N}_{\varepsilon^{2}}$ 
such that $\left\|z-z_{0}\right\| \leq \varepsilon^{2}$. Then

$$
\begin{aligned}
& \left\|\frac{1}{m} \sum_{k=1}^{m}\left|\boldsymbol{a}_{k}^{\top} \boldsymbol{z}\right|^{2} \boldsymbol{a}_{k} \boldsymbol{a}_{k}^{\top} \mathbf{1}_{\left\{\left|\boldsymbol{a}_{k}^{\top} \boldsymbol{z}\right| \leq R\right\}}-\frac{1}{m} \sum_{k=1}^{m}\left|\boldsymbol{a}_{k}^{\top} \boldsymbol{z}_{0}\right|^{2} \boldsymbol{a}_{k} \boldsymbol{a}_{k}^{\top} \mathbf{1}_{\left\{\left|\boldsymbol{a}_{k}^{\top} z_{0}\right| \leq R\right\}}\right\| \\
& \leq\left\|\frac{1}{m} \sum_{k=1}^{m}\left|\boldsymbol{a}_{k}^{\top} \boldsymbol{z}\right|^{2} \boldsymbol{a}_{k} \boldsymbol{a}_{k}^{\top} \mathbf{1}_{\left\{\left|\boldsymbol{a}_{k}^{\top} \boldsymbol{z}\right| \leq R\right\}} \mathbf{1}_{\left\{\left|\boldsymbol{a}_{k}^{\top} z_{0}\right|>R\right\}}\right\|+\left\|\frac{1}{m} \sum_{k=1}^{m}\left|\boldsymbol{a}_{k}^{\top} \boldsymbol{z}_{0}\right|^{2} \boldsymbol{a}_{k} \boldsymbol{a}_{k}^{\top} \mathbf{1}_{\left\{\left|\boldsymbol{a}_{k}^{\top} \boldsymbol{z}\right|>R\right\}} \mathbf{1}_{\left\{\left|\boldsymbol{a}_{k}^{\top} z_{0}\right| \leq R\right\}}\right\| \\
& +\left\|\frac{1}{m} \sum_{k=1}^{m}\left(\left|\boldsymbol{a}_{k}^{\top} \boldsymbol{z}\right|^{2}-\left|\boldsymbol{a}_{k}^{\top} \boldsymbol{z}_{0}\right|^{2}\right) \boldsymbol{a}_{k} \boldsymbol{a}_{k}^{\top} \mathbf{1}_{\left\{\left|\boldsymbol{a}_{k}^{\top} \boldsymbol{z}\right| \leq R\right\}} \mathbf{1}_{\left\{\left|\boldsymbol{a}_{k}^{\top} z_{0}\right| \leq R\right\}}\right\| \\
& \leq\left\|\frac{1}{m} \sum_{k=1}^{m}\left|\boldsymbol{a}_{k}^{\top} \boldsymbol{z}\right|^{2} \boldsymbol{a}_{k} \boldsymbol{a}_{k}^{\top} \mathbf{1}_{\left\{\left|\boldsymbol{a}_{k}^{\top} \boldsymbol{z}\right| \leq R\right\}} \mathbf{1}_{\left\{\left|\boldsymbol{a}_{k}^{\top} z_{0}\right|>R\right\}}\right\|+\left\|\frac{1}{m} \sum_{k=1}^{m}\left|\boldsymbol{a}_{k}^{\top} \boldsymbol{z}_{0}\right|^{2} \boldsymbol{a}_{k} \boldsymbol{a}_{k}^{\top} \mathbf{1}_{\left\{\left|\boldsymbol{a}_{k}^{\top} \boldsymbol{z}\right|>R\right\}} \mathbf{1}_{\left\{\left|\boldsymbol{a}_{k}^{\top} z_{0}\right| \leq R\right\}}\right\| \\
& +\left\|\frac{1}{m} \sum_{k=1}^{m}\left(\left|\boldsymbol{a}_{k}^{\top} \boldsymbol{z}\right|^{2}-\left|\boldsymbol{a}_{k}^{\top} \boldsymbol{z}_{0}\right|^{2}\right) \boldsymbol{a}_{k} \boldsymbol{a}_{k}^{\top} \mathbf{1}_{\left\{\left|\boldsymbol{a}_{k}^{\top} z\right| \leq R\right\}} \mathbf{1}_{\left\{\left|\boldsymbol{a}_{k}^{\top} z_{0}\right| \leq R\right\}} \mathbf{1}_{\left\{\left|\boldsymbol{a}_{k}^{\top}\left(z_{0}-\boldsymbol{z}\right)\right| \leq \varepsilon\right\}}\right\| \\
& +\left\|\frac{1}{m} \sum_{k=1}^{m}\left(\left|\boldsymbol{a}_{k}^{\top} \boldsymbol{z}\right|^{2}-\left|\boldsymbol{a}_{k}^{\top} \boldsymbol{z}_{0}\right|^{2}\right) \boldsymbol{a}_{k} \boldsymbol{a}_{k}^{\top} \mathbf{1}_{\left\{\left|\boldsymbol{a}_{k}^{\top} z\right| \leq R\right\}} \mathbf{1}_{\left\{\left|\boldsymbol{a}_{k}^{\top} z_{0}\right| \leq R\right\}} \mathbf{1}_{\left\{\left|\boldsymbol{a}_{k}^{\top}\left(z_{0}-\boldsymbol{z}\right)\right|>\varepsilon\right\}}\right\| \\
& \leq\left\|\frac{1}{m} \sum_{k=1}^{m}\left|\boldsymbol{a}_{k}^{\top} \boldsymbol{z}\right|^{2} \boldsymbol{a}_{k} \boldsymbol{a}_{k}^{\top} \mathbf{1}_{\left\{\left|\boldsymbol{a}_{k}^{\top} z\right| \leq R\right\}} \mathbf{1}_{\left\{\left|\boldsymbol{a}_{k}^{\top} z_{0}\right|>R\right\}}\right\|+\left\|\frac{1}{m} \sum_{k=1}^{m}\left|\boldsymbol{a}_{k}^{\top} \boldsymbol{z}_{0}\right|^{2} \boldsymbol{a}_{k} \boldsymbol{a}_{k}^{\top} \mathbf{1}_{\left\{\left|\boldsymbol{a}_{k}^{\top} \boldsymbol{z}\right|>R\right\}} \mathbf{1}_{\left\{\left|\boldsymbol{a}_{k}^{\top} z_{0}\right| \leq R\right\}}\right\| \\
& +\left\|\frac{1}{m} \sum_{k=1}^{m}\left(\left|\boldsymbol{a}_{k}^{\top} \boldsymbol{z}\right|^{2}-\left|\boldsymbol{a}_{k}^{\top} \boldsymbol{z}_{0}\right|^{2}\right) \boldsymbol{a}_{k} \boldsymbol{a}_{k}^{\top} \mathbf{1}_{\left\{\left|\boldsymbol{a}_{k}^{\top} z\right| \leq R\right\}} \mathbf{1}_{\left\{\left|\boldsymbol{a}_{k}^{\top} z_{0}\right| \leq R\right\}} \mathbf{1}_{\left\{\left|\boldsymbol{a}_{k}^{\top}\left(z_{0}-\boldsymbol{z}\right)\right| \leq \varepsilon\right\}}\right\| \\
& +\left\|\frac{1}{m} \sum_{k=1}^{m}\left(\left|\boldsymbol{a}_{k}^{\top} \boldsymbol{z}\right|^{2}-\left|\boldsymbol{a}_{k}^{\top} \boldsymbol{z}_{0}\right|^{2}\right) \boldsymbol{a}_{k} \boldsymbol{a}_{k}^{\top} \mathbf{1}_{\left\{\left|\boldsymbol{a}_{k}^{\top} z\right| \leq R\right\}} \mathbf{1}_{\left\{\left|\boldsymbol{a}_{k}^{\top} z_{0}\right| \leq R\right\}} \mathbf{1}_{\left\{\left|\boldsymbol{a}_{k}^{\top}\left(z_{0}-\boldsymbol{z}\right)\right|>\frac{\left\|z-z_{0}\right\|}{\varepsilon}\right\}}\right\| \\
& \leq R^{2}\left(\left\|\frac{1}{m} \sum_{k=1}^{m} \boldsymbol{a}_{k} \boldsymbol{a}_{k}^{\top} \mathbf{1}_{\left\{\left|\boldsymbol{a}_{k}^{\top} z_{0}\right|>R\right\}}\right\|+\left\|\frac{1}{m} \sum_{k=1}^{m} \boldsymbol{a}_{k} \boldsymbol{a}_{k}^{\top} \mathbf{1}_{\left\{\left|\boldsymbol{a}_{k}^{\top} \boldsymbol{z}\right|>R\right\}}\right\|\right) \\
& +2 R \boldsymbol{\varepsilon}\left\|\frac{1}{m} \sum_{k=1}^{m} \boldsymbol{a}_{k} \boldsymbol{a}_{k}^{\top}\right\|+2 R^{2}\left\|\frac{1}{m} \sum_{k=1}^{m} \boldsymbol{a}_{k} \boldsymbol{a}_{k}^{\top} \mathbf{1}_{\left\{\left|\boldsymbol{a}_{k}^{\top}\left(z_{0}-\boldsymbol{z}\right)\right|>\frac{\left\|z-z_{0}\right\|}{\varepsilon}\right\}}\right\| \\
& \leq 2 R^{2}\left(5 K^{2} R^{2} \exp \left(1-\frac{c_{1} R^{2}}{K^{2}}\right)+5 \frac{K^{2}}{\varepsilon^{2}} \exp \left(1-\frac{c_{1}}{K^{2} \varepsilon^{2}}\right)+2 \varepsilon\right)+4 R \varepsilon \text {. }
\end{aligned}
$$

The last inequality is according to Lemma 6.1 and Lemma 6.4. Based on (6.9) and (6.10), for any $\boldsymbol{z} \in \mathbb{S}^{n-1}$, we have

$$
\begin{aligned}
& \left\|\frac{1}{m} \sum_{k=1}^{m}\left|\boldsymbol{a}_{k}^{\top} \boldsymbol{z}\right|^{2} \boldsymbol{a}_{k} \boldsymbol{a}_{k}^{\top} \mathbf{1}_{\left\{\left|\boldsymbol{a}_{k}^{\top} \boldsymbol{z}\right| \leq R\right\}}-\left(2 z z^{\top}+\boldsymbol{I}+(\tau-3) \operatorname{Diag}\left(|z|^{2}\right)\right)\right\| \\
\leq & \left\|\frac{1}{m} \sum_{k=1}^{m}\left|\boldsymbol{a}_{k}^{\top} \boldsymbol{z}\right|^{2} \boldsymbol{a}_{k} \boldsymbol{a}_{k}^{\top} \mathbf{1}_{\left\{\left|\boldsymbol{a}_{k}^{\top} \boldsymbol{z}\right| \leq R\right\}}-\frac{1}{m} \sum_{k=1}^{m}\left|\boldsymbol{a}_{k}^{\top} \boldsymbol{z}_{0}\right|^{2} \boldsymbol{a}_{k} \boldsymbol{a}_{k}^{\top} \mathbf{1}_{\left\{\left|\boldsymbol{a}_{k}^{\top} z_{0}\right| \leq R\right\}}\right\|
\end{aligned}
$$




$$
\begin{aligned}
& +\left\|\frac{1}{m} \sum_{k=1}^{m}\left|\boldsymbol{a}_{k}^{\top} z_{0}\right|^{2} \boldsymbol{a}_{k} \boldsymbol{a}_{k}^{\top} \mathbf{1}_{\left\{\left|\boldsymbol{a}_{k}^{\top} z_{0}\right| \leq R\right\}}-\left(2 z_{0} z_{0}^{\top}+\boldsymbol{I}+(\tau-3) \operatorname{Diag}\left(\left|z_{0}\right|^{2}\right)\right)\right\| \\
& +\left\|\left(2 z_{0} z_{0}^{\top}+\boldsymbol{I}+(\tau-3) \operatorname{Diag}\left(\left|z_{0}\right|^{2}\right)\right)-\left(2 z z^{\top}+\boldsymbol{I}+(\tau-3) \operatorname{Diag}\left(|z|^{2}\right)\right)\right\| \\
\leq & 4 R \varepsilon+2 R^{2}\left(5 K^{2} R^{2} \exp \left(1-\frac{c_{1} R^{2}}{K^{2}}\right)+5 \frac{K^{2}}{\varepsilon^{2}} \exp \left(1-\frac{c_{1}}{K^{2} \varepsilon^{2}}\right)+2 \varepsilon\right) \\
& +\frac{1+R^{2} K^{2}}{2} \varepsilon+64 C^{4} K^{4} \exp \left(1-c_{0} R^{2} /(C K)^{2}\right)+4(1+|\tau-3|) \varepsilon^{2} \\
\leq & \left(5+4 R+5 R^{2} K^{2}\right) \varepsilon+\left(10 K^{2} R^{4}+64 C^{4} K^{4}\right) \exp \left(1-\frac{c R^{2}}{K^{2}}\right)+\frac{10 R^{2} K^{2}}{\varepsilon^{2}} \exp \left(1-\frac{c_{1}}{K^{2} \varepsilon^{2}}\right),
\end{aligned}
$$

provided $(1+|\tau-3|) \varepsilon<1$. From Lemma 6.1 and Lemma 6.4, one has

$$
\begin{aligned}
& \left\|\frac{1}{m} \sum_{k=1}^{m}\left|\boldsymbol{a}_{k}^{\top} \boldsymbol{z}\right|^{2} \boldsymbol{a}_{k} \boldsymbol{a}_{k}^{\top} \mathbf{1}_{\left\{\left\{\left|\boldsymbol{a}_{j}^{\top} z\right| \leq R\|\boldsymbol{z}\|_{2}\right\} \cap\left\{\sqrt{y_{j}} \leq R \sqrt{\frac{1}{m} \sum_{j=1}^{m} y_{i}}\right\}\right\}}-\left(2 z z^{\top}+\|z\|_{2}^{2} \boldsymbol{I}+(\tau-3) \operatorname{Diag}\left(|z|^{2}\right)\right)\right\| \\
\leq & \left\|\frac{1}{m} \sum_{k=1}^{m}\left|\boldsymbol{a}_{k}^{\top} \boldsymbol{z}\right|^{2} \boldsymbol{a}_{k} \boldsymbol{a}_{k}^{\top} \mathbf{1}_{\left\{\left|\boldsymbol{a}_{k}^{\top} \boldsymbol{z}\right| \leq R\right\}}-\left(2 \boldsymbol{z} \boldsymbol{z}^{\top}+\boldsymbol{I}+(\tau-3) \operatorname{Diag}\left(|\boldsymbol{z}|^{2}\right)\right)\right\| \\
& +\left\|\frac{1}{m} \sum_{k=1}^{m}\left|\boldsymbol{a}_{k}^{\top} \boldsymbol{z}\right|^{2} \boldsymbol{a}_{k} \boldsymbol{a}_{k}^{\top} \mathbf{1}_{\left\{\left\{\left|\boldsymbol{a}_{j}^{\top} z\right| \leq R\|z\|_{2}\right\} \cap\left\{\sqrt{y_{j}}>R \sqrt{\frac{1}{m} \sum_{j=1}^{m} y_{i}}\right\}\right\} \|}\right\| \\
\leq & \left(5+4 R+5 R^{2} K^{2}\right) \boldsymbol{\varepsilon}+\left(10 K^{2} R^{4}+64 C^{4} K^{4}\right) \exp \left(1-\frac{c R^{2}}{K^{2}}\right)+\frac{10 R^{2} K^{2}}{\varepsilon^{2}} \exp \left(1-\frac{c_{1}}{K^{2} \varepsilon^{2}}\right) \\
& +R^{2}\left(6 K^{2} R^{2} \exp \left(1-\frac{c_{1} R^{2}}{K^{2}}\right)+\varepsilon\right) \\
\leq & \left(5+4 R+6 R^{2} K^{2}\right) \varepsilon+\left(16 K^{2} R^{4}+64 C^{4} K^{4}\right) \exp \left(1-\frac{c R^{2}}{K^{2}}\right)+\frac{10 R^{2} K^{2}}{\varepsilon^{2}} \exp \left(1-\frac{c_{1}}{K^{2} \varepsilon^{2}}\right)
\end{aligned}
$$

for any $z \in \mathbb{S}^{n-1}$.

\section{Acknowledgments}

This work was supported by the NSAF of China under grant numbers 11901143,11971427, and 12101170, and the Zhejiang Provincial Natural Science Foundation of China under grant number LQ19A010008.

\section{REFERENCES}

[1] B. Gao, H.X. Liu, Y. Wang, Phase retrieval for sub-Gaussian measurements, Appl. Comput. Harmon. Anal. 53 (2021), 95-115.

[2] F. Kramher, Y. Liu, Phase retrieval without small-ball probability assumptions, IEEE Trans. Inform. Theory, 64 (2018), 485-500.

[3] D. Suess, R. Kueng, D. Gross, Certifying linear optical circuits via phaseless estimation techniques, Conference on Quantum Info. Process. (2016), 10-15.

[4] S. Foucart, H. Rauhut, A Mathematical Introduction to Compressive Sensing, Birkhauser, Boston, 2013. 
[5] R. Vershynin, Introduction to the non-asymptotic analysis of random matrices. arXiv:1011.3027, 2010.

[6] J.F. Cai, K. Wei, Solving systems of phaseless equations via Riemannian optimization with optimal sampling complexity, arXiv:1809.02773, 2018.

[7] K. Wei, J.F. Cai, T.F. Chan, S. Leung, Guarantees of Riemannian optimization for low rank matrix recovery, SIAM J. Matrix Anal. Appl. 37 (2016),1198-1222.

[8] A.S. Bandeira, Y. Chen, D.G. Mixon, Phase retrieval from power spectra of masked signals, Inform. Inference, 3 (2014), 83-102.

[9] A. Chai, M. Moscoso, G. Papanicolaou, Array imaging using intensity-only measurements, Inverse Probl. 27 (2011), 015005.

[10] J.V. Corbett, The pauli problem, state reconstruction and quantum-real numbers, Rep. Math. Phys. 57 (2006), 53-68.

[11] J.C. Dainty, J.R. Fienup, Array imaging using intensity-only measurements, phase retrieval and image reconstruction for astronomy, In: H. Stark (Ed), Image Recovery: Theory and Application, pp. 231-275, Academic Press, San Diego, 1987.

[12] R.W. Harrison, Phase problem in crystallography, J. Opt. Soc. Amer. A 10 (1993), 1046-1055.

[13] R.Millane, Phase retrieval in crystallography and optics, J. Opt. Soc. Amer. A 7 (1990), 394-411.

[14] R.W. Gerchberg, W.O. Saxton, Practical algorithm for the determination of phase from image and diffraction plane pictures, Optik 35 (1972), 237-246.

[15] J.R. Fienup, Phase retrieval algorithms: a comparison, Appl. Opt. 21 (1982), 2758-2769.

[16] Y. Shechtman, Y.C. Eldar, O. Cohen, H N. Chapman, J. Miao, M. Segev, Phase retrieval with application to optical imaging, IEEE Signal Processing Mag. 32 (2015), 87-109.

[17] E.J. Candès, Y.C. Eldar, T. Strohmer, V. Voroninshi, Phase retrieval via matrix completion, SIAM J. Imaging Sci. 6 (2013), 199-225.

[18] E.J. Candès, X. Li, Solving quadratic equations via phaselift when there are about as many equations as unknowns, Found. Comput. Math. 149 (2013), 1-10.

[19] E.J. Candès, T. Strohmer, V. Voroninshi, PhaseLift: exact and stable signal recovery from magnitude measurements via convex programming, Comm. Pure Appl. Math. 66 (2013), 1241-1274.

[20] R. Kueng, H. Rauhut, U. Terstiege, Low rank matrix recovery from rank one measurements, Appl. Comput. Harmon. Anal. 42 (2014), 88-116.

[21] E.J. Candès, X. Li, M. Soltanolkotabi, Phase retrieval via Wirtinger flow: theory and algorithms, IEEE Trans. Inform. Theory 61 (2015), 1985-2007.

[22] P. Netrapalli, P. Jain, S. Sanghavi, Phase retrieval using alternating minimization, IEEE Trans. Signal Process. 63 (2015), 4814-4826.

[23] Y. Tan, R. Vershynin, Phase retrieval via randomized Kaczmarz: theoretical guarantees, Information and Inference 8 (2019), 97-123.

[24] G. Wang, G.B. Giannakis, J. Chen, Scalable solvers of random quadratic equations via stochastic truncated amplitude flow, IEEE Trans. Signal Process. 65 (2017), 1961-1974.

[25] G. Wang, G.B. Giannakis, Y.C. Eldar, Solving systems of random quadratic equations via truncated amplitude flow, IEEE Trans. Inform. Theory 64 (2018), 773-794. 